\title{
SOME RENEWAL THEOREMS FOR NON-NEGATIVE INDEPENDENT RANDOM VARIABLES
}

\author{
JOHN A. WILLIAMSON ${ }^{1}$ )
}

Introduction.

Definition. Let $\left\{X_{n}\right\}$ be a sequence of random variables with $S_{n}=$ $\sum_{k=1}^{n} X_{k}$. Denote by $I_{k}(t)$ the indicator function of the event $\left\{S_{k}<t\right\}$. The random variable $N(t)$ is defined by

$$
N(t)=\sum_{k=1}^{\infty} I_{k}(t)
$$

Renewal theory is concerned with the random variable $N(t)$, with random variables defined in terms of $N(t)$, and with the asymptotic behavior of the moments and distributions of these random variables. In this paper we examine, under certain conditions, the behavior of $E(N(t))$ as $t$ becomes large. Hence in the introduction we will mention only results in this general area.

$$
E(N(t))=\sum_{k=1}^{\infty} E\left(I_{k}(t)\right)=\sum_{k=1}^{\infty} P\left(S_{k}<t\right) .
$$

We set $H(t)=E(N(t))$ and call $H(t)$ the renewal function.

Feller [7] in 1941 was able to show that for positive, independent, identically distributed random variables,

$$
\lim _{x \rightarrow \infty} H(x) / x=1 / E\left(X_{1}\right)
$$

Definition. Let $\left\{X_{n}\right\}$ be a sequence of random variables. If there exists no real number, $d$, for which for all $n, \sum_{k=-\infty}^{\infty} P\left(X_{n}=k d\right)=1$, then we will say that $\left\{X_{n}\right\}$ is a continuous renewal sequence.

In 1948, Blackwell [1] proved that if $\left\{X_{n}\right\}$ is a continuous renewal sequence of non-negative independent, identically distributed, random variables, then for any $h>0, \lim _{x \rightarrow \infty}(H(x)-H(x-h))=h / E\left(X_{1}\right)$.

Definition. For a sequence $\left\{X_{n}\right\}$ of integer valued random variables, we let

$$
u_{n}=\sum_{k=1}^{\infty} P\left(\sum_{j=1}^{k} X_{j}=n\right)=H(n+1)-H(n)
$$

Presented to the Society, January 28, 1963; received by the editors August 12, 1963.

( $\left.{ }^{1}\right)$ This research was partially supported by the Air Force Office of Scientific Research and partially by a fellowship from the National Aeronautics and Space Administration. 
If the random variables are positive, then $u_{n}$ can be interpreted as the probability of a renewal at time $n$. If $\left\{X_{n}\right\}$ is a sequence of positive, integer valued, independent, identically distributed, random variables, then as a consequence of a 1949 theorem by Erdös, Feller, and Pollard [6], $\lim _{n \rightarrow \infty} u_{n}=$ $1 / E\left(X_{1}\right)$. This result also follows immediately from the much earlier ergodic theorem for Markov chains proven in 1936 by Kolmogorov [11]. In the above results $1 / E\left(X_{1}\right)$ is to be interpreted as 0 if $E\left(X_{1}\right)=\infty$.

These last two results for continuous, and integer valued renewal sequences might be called exact renewal theorems. Generalizations of these results have taken two general directions.

Chung and Wolfowitz [4] in 1952 and Blackwell [2] in 1953 obtained exact renewal theorems for integer valued, and continuous renewal sequences respectively for the case when the random variables are independent, identically distributed and $0<E\left(X_{1}\right) \leqq \infty$. Feller and Orey [8] in 1961 obtained results for $\lim _{x \rightarrow \infty}(H(x)-H(x-h))$ where it was only assumed that the random variables of the continuous renewal sequences were independent and identically distributed.

If it is not required that the random variables be identically distributed, then it is still possible to obtain results. Kawata [10] in 1956 proved that, under conditions, for any $h>0, \lim _{x \rightarrow \infty}(1 / x) \int_{0}^{x}(H(y)-H(y-h)) d y=h / \alpha$, and in 1963 Chow and Robbins [3] showed that under similar conditions, $\lim _{x \rightarrow \infty} H(x) / x=1 / \alpha$. In each of these Cesaro renewal theorems: $\alpha=$ $\lim _{n \rightarrow \infty}(1 / n) \sum_{k=1}^{n} E\left(X_{k}\right)$, the variables are assumed to be independent, and restrictions are placed on the tails of the distribution functions of the random variables.

Smith [12] in 1961 was able to prove an exact renewal theorem for sequences of independent, but not necessarily identically distributed, random variables. It is toward this same end that the work of this paper is directed.

In the following pages only non-negative random variables are considered. Actually the results of the first two sections, with the exception of Lemma 2 -A, can be proven without restricting the random variables to non-negative values. The proofs are the same with only the limits on some integrals and sums having to be changed. However in the proof of Theorem 3 and its corollaries the non-negative assumption is needed, so we also write out the earlier proofs under this rather natural assumption. None of Smith's results require that the random variables be non-negative.

In both Smith's paper and $\S 2$ of this paper conditions are sought which rule out the possibility that for some $h>0, H(x)-H(x-h)$, or an approximation to it, will behave periodically as $x$ becomes large. In $\S 1$ of this paper the integer valued case is similarly treated. The strength of the theorems and corollaries of $\S \S 1$ and 2 lies in the fact that their conclusions follow from the 
existence of a subsequence with certain properties rather than from the negation of the existence of subsequences with certain properties as is the case in Smith's comparable results. Furthermore, the way in which the subsequence is distributed through the original sequence is not a factor. In Smith's insistent mesh restrictions the distribution of subsequences is important.

Lemma 3-B with Theorem 3 and Corollary 3-A imposes the same conditions on the tails of the distribution functions of the random variables as do Smith's Theorems 6, 7, and 8. Lemmas 3-C and 3-D extend the application of Theorem 3 to sequences of random variables which, because of the behavior of the tails of their distribution functions, do not lie in the domain of Smith's theorems. There are certain difficulties which we have not been able to surmount. Smith's successes with them are discussed briefly in the last paragraph of $\$ 2$.

Throughout these pages the following notation will be used.

By $\left\{f_{k}\right\} *\left\{g_{k}\right\}$ will be meant the sequence $\left\{h_{k}\right\}$ where

$$
h_{k}=\sum_{j=-\infty}^{\infty} f_{j} g_{k-j}
$$

By $F * G(x)$ will be meant $F * G(x)=\int F(x-y) d G(y)$.

The expression, $[y]$, where it appears as a limit of a summation should be read as the greatest integer in $y$.

I would like to thank Professor Steven Orey of the Department of Mathematics of the University of Minnesota, under whose direction this work was done. I would also like to thank Professor Harry Furstenberg of the same department for a key suggestion.

Added in proof. The following unpublished result by $\mathrm{H}$. Kesten was brought to our attention after the completion of this paper. If the conditions (a), (b), (c) of Theorem 7 in Smith [12] are satisfied and if for every $\epsilon_{0}>0$ and integer $B>0$ there exists $\epsilon_{1}>0$ such that for any $k$ it is possible to find $X_{i_{1}}, \cdots, X_{i_{r}}$ with all $i_{j} \geqq k$ and $C$ satisfying

$$
p\left\{\sum_{j=1}^{r} X_{i_{j}} \in\left(C+(s-1) \epsilon_{0}, C+s \epsilon_{0}\right)\right\} \geqq \epsilon_{1}
$$

for $s=1,2, \cdots, B$ then $\lim _{x \rightarrow \infty}[H(x)-H(x-h)]=h / \mu$. This result is partly contained in and partly complements $\$ \S 2$ and 3 of this paper.

1. Conditions which insure that $\lim _{n \rightarrow \infty}\left(u_{n}-u_{n-1}\right)=0$. It is the purpose of this section to establish conditions under which $\lim _{n \rightarrow \infty}\left(u_{n}-u_{n-1}\right)=0$. Although the principal concern in this section is with sequences of nonnegative, independent, integer valued, random variables, the hypotheses in Lemmas 1-A and 1-B will be weakened so that their conclusions will also be of use in the discussion of continuous renewal sequences in $\$ 2$. 
LEMMA 1-A. Let $\left\{X_{k}\right\}$ be a sequence of random variables with $\phi_{k}(t)=E\left(e^{i t X_{k}}\right)$. If there exist a sequence $\left\{a_{k}\right\}$ of real numbers and a positive constant, $M$, such that:

(1.1) there exists some $\omega>0$ for which $P\left(\left|X_{k}-a_{k}\right|<M\right) \geqq \omega$ for all $k$;

(1.2) there exists some $c>0$ for which

$$
\begin{aligned}
E\left\{\left[X_{k}-E\left(X_{k}|| X_{k}-a_{k} \mid\right.\right.\right. & \left.<M)]^{2}|| X_{k}-a_{k} \mid<M\right\} \\
& =\operatorname{var}\left(X_{k}|| X_{k}-a_{k} \mid<M\right) \geqq c
\end{aligned}
$$

for all $k$; then there exist constants $b>0$ and $d>0$ such that if $|t|<d$ then for all $k,\left|\phi_{k}(t)\right| \leqq 1-b t^{2}$.

Proof. We consider first the situation where, for all $k, P\left(\left|X_{k}-a_{k}\right|<M\right)=1$. In this case, of course, $\omega$ may be taken to be 1 . Set $d=1 / 4 M$ and $b=c / 3$. The result for this case follows from a known result stated as 11.14 in Doob $[5$, p. 45].

In the case where we have (1.1) for some $\omega, 0<\omega<1$, we can write

$$
\begin{aligned}
& \left|\phi_{k}(t)\right| \leqq\left|\int_{\left|x-a_{k}\right| \geqq M} e^{i t x} d P\left(X_{k}<x\right)+\int_{\left|x-a_{k}\right|<M} e^{i t x} d P\left(X_{k}<x\right)\right| \\
& \quad \leqq \int_{\left|x-a_{k}\right| \geqq M} d P\left(X_{k}<x\right)+P\left(\left|X_{k}-a_{k}\right|<M\right)\left|\int_{\left|x-a_{k}\right|<M} \frac{e^{i t x} d P\left(X_{k}<x\right)}{P\left(\left|X_{k}-a_{k}\right|<M\right)}\right| .
\end{aligned}
$$

Therefore if $|t| \leqq d$ then

$$
\left|\phi_{k}(t)\right| \leqq P\left(\left|X_{k}-a_{k}\right| \geqq M\right)+P\left(\left|X_{k}-a_{k}\right|<M\right)\left(1-b t^{2}\right) \leqq 1-\omega b t^{2} .
$$

Absorbing $\omega$ into the constant $b$ completes the proof.

A statement like Lemma 1-A about the uniform behavior near 0 of a sequence of moduli of characteristic functions is of some intrinsic interest. The following lemma is designed to meet the specific needs of the main theorem of this section.

Definition. Let $F(x)$ be a function of bounded variation on $(-\infty, \infty)$. By $\|F\|$ we shall mean

(1.3) $\|F\|=S|d F(x)|=$ total variation of $F$ on $(-\infty, \infty)$.

Lемма 1-B. Let $\left\{F_{k}\right\},\left\{L_{k}\right\}$, and $\left\{K_{k}\right\}$ be sequences of probability distributions and $U$ a function of bounded variation on $(-\infty, \infty)$. If we can write $F_{k}(x)=\nu_{k} L_{k}(x)+\left(1-\nu_{k}\right) K_{k}(x)$ where $0<\omega \leqq \nu_{k} \leqq 1$ for all $k$ and some $\omega$, and if $\lim _{n \rightarrow \infty}\left\|\left(*_{k=1}^{n} L_{j_{k}}\right) * U\right\|=0$ uniformly for all sequences $\left\{j_{k}\right\}$, then

$$
\lim _{n \rightarrow \infty}\left\|\left(\begin{array}{c}
n \\
* \\
k=1
\end{array} F_{k}\right) * U\right\|=0 .
$$

Proof. Let $\nu>0$ be given. Choose $N^{\prime}$ so that for all $n \geqq N^{\prime},\left\|\left(*_{k=1}^{n} L_{j_{k}}\right) * U\right\|$ $\leqq \nu / 2$, for all sequences $\left\{j_{k}\right\}$. Using the associative, commutative, and distributive properties of the convolution operation, we can expand 
$\left\|\left(\underset{k=1}{*} F_{k}\right) * U\right\|=\left\|\left[\begin{array}{c}n \\ * \\ k=1\end{array}\left(\nu_{k} L_{k}+\left[1-\nu_{k}\right] K_{k}\right)\right] * U\right\| \leqq\left(\prod_{k=1}^{n} \nu_{k}\right)\left\|\left(\begin{array}{c}n \\ * \\ k=1\end{array} L_{k}\right) * U\right\|$

$+\sum_{j=1}^{n}\left(\prod_{k=1 ; k \neq j}^{n} \nu_{k}\right)\left(1-\nu_{j}\right)\left\|\left(\begin{array}{cc}n \\ * & L_{k}\end{array}\right) * K_{j} * U\right\|$

$+\sum_{i=1 ; i>j ;}^{n} \sum_{j=1}^{n}\left(\prod_{k=1 ; k \neq i ; k \neq j}^{n} \nu_{k}\right)\left(1-\nu_{j}\right)\left(1-\nu_{i}\right)\left\|\left(\underset{k=1 ; k \neq j ; k \neq i}{*} L_{k}\right) * K_{j} * K_{i} * U\right\|+\cdots$

$+\sum_{j=1}^{n}\left(\prod_{k=1 ; k \neq j}^{n}\left[1-\nu_{k}\right] \nu_{j}\right)\left\|\left(\begin{array}{c}n \\ * \\ k=1: k \neq j\end{array} K_{k}\right) * L_{j} * U\right\|$

$+\left(\prod_{k=1}^{n}\left[1-v_{k}\right]\right)\left\|\left(\underset{k=1}{*} K_{k}\right) * U\right\|$.

For each $k,\left\|K_{k}\right\| \leqq 1$ so that each expression which contains $N^{\prime}$ or more of the $L_{k}$ can be estimated $\nu / 2$ times the appropriate product of the $\nu_{j}$ and $\left(1-\nu_{j}\right)$. If $n>2 N^{\prime}$ then the sum of the remaining terms is less than or equal to

$$
\left(N^{\prime}\right)\left(\begin{array}{c}
n \\
N^{\prime}-1
\end{array}\right)(1-\omega)^{n-N^{\prime}+1}\|U\|
$$

But this quantity can be made less than $\nu / 2$ for all $n$ greater than some $N^{\prime \prime}>2 N^{\prime}$. Therefore, for all $n>N^{\prime \prime},\left\|\left(*_{k=1}^{n} F_{k}\right) * U\right\| \leqq(\nu / 2) \prod_{k=1}^{n}\left(\nu_{k}+\left[1-\nu_{k}\right]\right)$ $+\nu / 2=\nu$. This gives the desired result.

This brings us to the principal result in this section.

Theorem 1. Let $\left\{X_{n}\right\}$ be a sequence of non-negative, independent, integer valued, random variables. If there exists a subsequence $\left\{X_{n_{k}}\right\}$ of $\left\{X_{n}\right\}$, a sequence $\left\{a_{k}\right\}$ of real numbers, and positive constants $M$ and $\omega$ such that:

(A) for all $k, P\left(\left|X_{n k}-a_{k}\right|<M\right) \geqq \omega$;

(B) for any $d, 0<d<\pi$, there exists $u>0, u=u(d)$, for which

$$
\sup _{k \geqq 1}\left|\rho_{k}(t)\right| \leqq 1-u
$$

for all $t$ satisfying $d \leqq|t| \leqq \pi$ where $\rho_{k}(t)=\int e^{i t x} d P\left(X_{n_{k}}<x|| X_{n_{k}}-a_{k} \mid<M\right)$;

(C) there exists some positive constant, $B$, such that for all $n$ and all $j$,

$$
\sum_{k=j}^{\infty} P\left(\sum_{i=j}^{k} X_{i}=n\right) \leqq B
$$

then $\lim _{n \rightarrow \infty}\left(u_{n}-u_{n-1}\right)=0$.

Proof. First it will be established that under the hypotheses of this theorem it is possible to apply Lemma 1-A to the subsequence $\left\{X_{n_{k}}\right\}$. Since the variance of a random variable and the absolute value of its characteristic function are both invariant under translation, it is sufficient to consider the case 
when all $a_{k}$ are 0 . To simplify the notation we introduce the random variables, $Y_{k}$, defined by $P\left(Y_{k}=m\right)=P\left(X_{n_{k}}=m|| X_{n_{k}}-a_{k} \mid<M\right)$. To show (1.4) implies (1.2) we assume that $\inf _{k \geqq 1}\left\{\operatorname{var}\left(Y_{k}\right)\right\}=0$. If each $a_{k}=0$ then $E\left(Y_{k}\right) \leqq M$ for all $k$ and hence there exists a subsequence $\left\{k_{j}\right\}$ such that

$$
\lim _{j \rightarrow \infty} \operatorname{var}\left(Y_{k_{j}}\right)=0 \text { and } \lim _{j \rightarrow \infty} E\left(Y_{k_{j}}\right)=m \leqq M
$$

But this means that the distribution function of $Y_{k_{j}}$ converges to the distribution function which assigns probability 1 to the point $m$ at every continuity point of the limiting distribution. Therefore $\lim _{j \rightarrow \infty} \rho_{k j}(t)=e^{i t m}$. However this contradicts the assumptions made in condition (B).

Next we introduce the following notation. Let $P_{j}(q)=P\left(S_{q}=j\right)$ and $u_{n}(k)$ $=\sum_{j=0}^{\infty} P\left(S_{k+j}-S_{k}=n\right)$. Then $u_{n}(0)=u_{n}$. If we let $u(k)=\left\{u_{n}(k)\right\}$ and $p(q)$ $=\left\{p_{j}(q)\right\}$, then $u_{n}$ can be expressed by $u_{n}=p_{n}(1)+p_{n}(2)+\cdots+p_{n}(q-1)$ $+\sum_{j=0}^{n} u_{n-j}(q) p_{j}(q)$. Denoting the $n$th term of the sequence $u(q) * p(q)$ by $u(q) * p(q)_{n}$ permits us to write

$$
u_{n}-u_{n-1}=\sum_{j=1}^{q-1}\left(p_{n}(j)-p_{n-1}(j)\right)+u(q) * p(q)_{n}-u(q) * p(q)_{n-1} .
$$

Since for each fixed $j, \lim _{n \rightarrow \infty}\left(p_{n}(j)-p_{n-1}(j)\right)=0$, the problem of showing that $\lim _{n \rightarrow \infty}\left(u_{n}-u_{n-1}\right)=0$ reduces to showing that

$$
\left|u(q) * p(q)_{n}-u(q) * p(q)_{n-1}\right|
$$

can be made arbitrarily small by taking $q$ large uniformly in $n$.

Case 1. Assume that for all $k, P\left(\left|X_{n_{k}}-a_{k}\right|<M\right)=1$. Since convolution is an associative operation, we can write $u(q) * p(q)$ as $v(q) * r(q)$ where $r_{j}(q)$ $=P\left(\sum_{k=1}^{q^{\prime}} X_{n_{k}}=j\right), q^{\prime}$ is the largest $n_{k}$ less than or equal to $q$, and $v(q)$ is the sequence formed by convolving $u(q)$ with the distributions of these $X_{n}$ for which $n$ is not equal to any $n_{k}$ and $n \leqq q$. (1.5) can then be written as $v(q) * r(q)_{n}-v(q) * r(q)_{n-1}$. But for all $j$ and $q,\left|v_{j}(q)\right| \leqq B$ so $(1.5)=$

$$
\left|\sum_{j=0}^{n} v_{n-j}(q) r_{j}(q)-\sum_{j=0}^{n-1} v_{n-1-j}(q) r_{j}(q)\right| \leqq B \sum_{j=0}^{\infty}\left|r_{j}(q)-r_{j-1}(q)\right| .
$$

Now let $\nu>0$ be given. By Lemma 1-A there exist positive numbers $b$ and $d$ such that for all $k$ and all $|t| \leqq d,\left|\rho_{k}(t)\right| \leqq 1-b t^{2}$. As a result of condition (B) of this theorem we can find $u>0$ which depends only on $d$ and for which $\left|\rho_{k}(t)\right| \leqq 1-u$ for all $k$ and all $t$ satisfying $d \leqq|t| \leqq \pi$. Choose $Q$ so large that the following inequalities are all satisfied for all $q \geqq Q$ :

$$
1 / b q^{\prime} \leqq \nu / 12 ; \quad\left(3+3 \nu b q^{\prime}\right)(1-u)^{q^{\prime}} \leqq \nu / 3 ;
$$

and

$$
P\left(\left|\sum_{k=1}^{q^{\prime}}\left(X_{n_{k}}-E\left(X_{n_{k}}\right)\right)\right| \geqq \nu b q^{\prime}\right) \leqq q^{\prime} M^{2} /\left(\nu b q^{\prime}\right)^{2} \leqq \nu / 12 .
$$


Under Case 1, $E\left(\exp i t X_{n_{k}}\right)=\rho_{k}(t)$ and $E\left(Y_{k}\right)=E\left(X_{n_{k}}\right)$ so that for all $q \geqq Q$,

$$
\begin{aligned}
& \sum_{j=0}^{\infty}\left|r_{j}(q)-r_{j-1}(q)\right|
\end{aligned}
$$

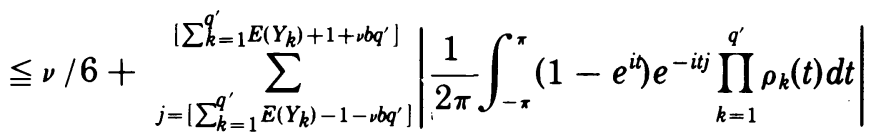

$$
\begin{aligned}
& \leqq \nu / 6+\frac{\left(3+2 q^{\prime} \nu b\right)}{\pi} \int_{0}^{d}\left(1-b t^{9}\right) t d t+\left(3+2 b \nu q^{\prime}\right)(1-u)^{q^{\prime}} \\
& \leqq \nu / 6+1 / 2 b q^{\prime}+\nu / \pi+\nu / 3 \leqq \nu .
\end{aligned}
$$

This completes the proof under the assumption of Case 1.

Case 2. If we have condition (A) only for some $\omega, 0<\omega<1$, then $\sum_{j=0}^{\infty}\left|r_{j}(q)-r_{j-1}(q)\right|$ can be written as $\left\|\left(*_{k=1}^{q^{\prime}} F_{k}\right) * U\right\|=$

$$
\|\left[\begin{array}{l}
q^{\prime} \\
\left.*\left(\nu_{k} L_{k}+\left[1-\nu_{k}\right] K_{k}\right)\right] * U \|
\end{array}\right.
$$

where

$$
\begin{array}{ll}
F_{k}(x)=P\left(X_{n_{k}}<x\right), & L_{k}(x)=P\left(X_{n_{k}}<x|| X_{n_{k}}\left|a_{k}\right|<M\right), \\
P\left(\left|X_{n_{k}}-a_{k}\right|<M\right), & K_{k}(x)=\left(F_{k}(x)-\nu_{k} L_{k}(x)\right) /\left(1-\nu_{k}\right),
\end{array}
$$

$U$ assigns weight -1 to point 1 and weight 1 to the point 0 , and the norm of the convolution of measures is to be interpreted as in (1.3). We have $\nu_{k} \geqq \omega>0$ for all $k$. From the Case 1 discussion it is seen that $\left\|\left(*_{k=1}^{n} L_{j_{k}}\right) * U\right\|$ can be made arbitrarily small for all sufficiently large $n$ uniformly for all sequences $\left\{j_{k}\right\}$ because the estimation based on the weak law depends only on $M$ and the number of terms, $n$, in the sequence, while the bounds on the $\left|\rho_{k}(t)\right|$ are uniform in $k$. Therefore we can apply Lemma 1-B to (1.6) and this proves the theorem.

REMARK. If all the random variables are strictly positive then condition (C) of Theorem 1 is satisfied with $B=1$. Another assumption which implies condition $(\mathrm{C})$ is discussed in Lemma 2-A.

Corollary 1-A. Let $\left\{X_{n}\right\}$ be a sequence of independent, integer valued, nonnegative, random variables. If there exists an identically distributed subsequence $\left\{X_{n_{k}}\right\}$ of $\left\{X_{n}\right\}$ with $\left|E\left(\exp i t X_{n_{1}}\right)\right|<1$ for each $t, 0<|t| \leqq \pi$, and if in addition condition (C) of Theorem 1 is satisfied, then $\lim _{n \rightarrow \infty}\left(u_{n}-u_{n-1}\right)=0$.

Proof. The positive constants $M$ and $\omega$ of Theorem 1 can obviously be found.

CoROLLARY 1-B. Let $\left\{X_{n}\right\}$ be a sequence of non-negative, independent, integer valued, random variables. If there exist a subsequence $\left\{X_{n_{k}}\right\}$ of $\left\{X_{n}\right\}$ and a positive constant s such that for each $k$ there exists $j=j(k)$ for which $P\left(X_{n_{k}}=j\right) \geqq s$ 
and $P\left(X_{n_{k}}=j+1\right) \geqq s$, and if condition (C) of Theorem 1 is satisfied, then $\lim _{n \rightarrow \infty}\left(u_{n}-u_{n-1}\right)=0$.

Proof. Let $P\left(x_{n_{k}}=j\right)=f_{j}(k)$. Using the notation of Theorem 1 let $a_{k}=$ $j(k)[j(k)+1] / 2, M=1$, and $\omega=2 s$. For all $k$ and all $|t| \leqq \pi / 2,\left|\rho_{k}(t)\right|$ $\leqq 1-4 s^{2} t^{2} / \pi^{2}$. For all $k$ and all $t$ satisfying $\pi / 2 \leqq|t| \leqq \pi,\left|\rho_{k}(t)\right| \leqq 1-s^{2}$. Conditions (A) and (B) of Theorem 1 are easily verified.

Corollaries more general than Corollary 1-B can be stated by not placing the restricting condition on consecutive integers. However, Theorem 1 does not allow us to conclude anything about the asymptotic behavior of $u_{n}-u_{n-1}$ from the presence of random variables which take on only values $(m+k d)$ where $k=1,2, \cdots, d \geqq 2$, and $m$ and $d$ are integers. Example 4.1 shows that the existence of a subsequence, $\left\{X_{n_{k}}\right\}$ for which for all $k, \sum_{j=0}^{\infty} P\left(X_{n_{k}}=m+j d\right)$ $=1, d \geqq 2$, is not enough to insure $\lim _{n \rightarrow \infty}\left(u_{n}-u_{n-1}\right)=0$ even when $\operatorname{gcd}(m, d)$ $=1$. Before continuing our discussion of $u_{n}-u_{n-1}$ there is another conclusion to be drawn from Theorem 1 .

Corollary 1-C. Let $\left\{X_{n}\right\}$ be a sequence of independent, non-negative, integer valued random variables. If there exist a subsequence $\left\{X_{n_{k}}\right\}$ of $\left\{X_{n}\right\}$, a sequence $\left\{a_{k}\right\}$ of real numbers, positive constants $M$ and $\omega$, and a positive integer $d$ such that:

(i) Forall $k, P\left(\left|X_{n_{k}}-a_{k}\right|<M\right) \geqq \omega$;

(ii) For any $h$ in $(-\pi / d, \pi / d)$ there exists $u>0, u=u(h)$, for which $\sup _{k \geqq 1}\left|\rho_{k}(t)\right| \leqq 1-u$ for all $t$ satisfying $h \leqq|t| \leqq \pi / d$ where $\rho_{k}(t)$ is defined as in Theorem 1;

(iii) Condition (C) of Theorem 1 is satisfied;

then $\lim _{n \rightarrow \infty}\left(u_{n}-u_{n-d}\right)=0$.

Proof. In the notation of Theorem 1 we need only show that

$$
\lim _{q \rightarrow \infty} \sum_{j=-\infty}^{\infty}\left|r_{j}(q)-r_{j-d}(q)\right|=0
$$

But

$$
r_{j}(q)-r_{j-d}(q)=(d / 2 \pi) \int_{-\pi / d}^{\pi / d} e^{-i j t}\left(1-e^{i d t}\right) \prod_{k=1}^{q^{\prime}} \rho_{k}(t) d t
$$

and the use on the interval $[-\pi / d, \pi / d]$ of the estimates made in Theorem 1 gives us the conclusion.

However, for $d \geqq 2$, Corollary $1-\mathrm{C}$ is of no help in establishing the main result, Theorem 3 , of $\S 3$.

For a sequence of random variables that take on only integer values of the form $m+k d, k=1,2, \cdots$, where $\operatorname{gcd}(m, d)=1$, stringent conditions can be stated which imply that $\lim _{n \rightarrow \infty}\left(u_{n}-u_{n-1}\right)=0$. If we are willing to impose the conditions of $\S 3$ in addition to those of this section, then we can obtain a more acceptable result. Corollary 3 -B states that if there exists some $N>0$ such that 
for all $n \geqq N, \sum_{j=0}^{\infty} P\left(X_{n}=m+j d\right)=1$ where $\operatorname{gcd}(m, d)=1$, and if for some subsequence conditions (i) and (ii) of Corollary 1-C are satisfied, then $u_{n}$ actually approaches a limit as $n$ approaches infinity provided that the hypotheses of the renewal theorem, Theorem 3, are also satisfied.

The particular case where the variables are identically distributed with $\sum_{k=0}^{\infty} P\left(X_{1}=m+k d\right)=1, d \geqq 2$, and $\operatorname{gcd}(m, d)=1$ can now be dealt with. The case when $E\left(X_{1}\right)$ is finite is handled by Corollary 3-B. If the first moment is infinite then Corollary 1-C can be combined with Corollary 3-A to get that $u_{n}$ approaches 0 as $n$ approaches infinity.

We conclude this section with what might be called a catch-all lemma. Unfortunately its condition, except in the identically distributed case, is almost impossible to verify.

Lемма 1-C. Let $\left\{X_{n}\right\}$ be a sequence of independent, integer valued, positive, random variables with $\phi_{k}(t)=E\left(e^{i t X_{k}}\right)$. If the function

$$
f(t)=\left(1-e^{i t}\right) \sum_{k=1}^{\infty}\left(\prod_{j=1}^{k} \phi_{j}(t)\right)
$$

is in $\mathscr{L}_{1}(-\pi, \pi)$ and $u_{n}$ is finite for each $n$, then $\lim _{n \rightarrow \infty}\left(u_{n}-u_{n-1}\right)=0$.

Proof. We can write

$$
u_{n}-u_{n-1}=(1 / 2 \pi) \int_{-\pi}^{\pi} e^{-i n t} f(t) d t
$$

and the result follows from the Riemann-Lebesgue lemma.

2.Conditions which insure that for any $h$ and certain $g(x)$,

$$
\lim _{x \rightarrow \infty} g *[H(x+h)-H(x)]=0 .
$$

In this section results analogous to those obtained in the first section will be established but without the restriction that the random variables be integer valued.

Definition. Let $\left\{X_{n}\right\}$ be a sequence of non-negative independent random variables with $S_{n}=\sum_{k=1}^{n} X_{k}$. Let $I_{k}(j, x)$ be the indicator function of the event $\left\{S_{k}-S_{j}<x\right\}$. The random variable $N_{j}(x)$ is defined by $N_{j}(x)=$ $\sum_{k=j+1}^{\infty} I_{k}(j, x)$. We will write $N(x)$ for $N_{0}(x)$ and set $H_{k}(x)=E\left(N_{k}(x)\right)=$ $\sum_{j=1}^{\infty}\left(*_{n=k+1}^{k+j} P\left(X_{n}<x\right)\right)$. For $H_{0}(x)$ we write $H(x)$.

In the main theorem of this section we will require that

(2.1) for any $z$ there exists a positive bound, $B(z)$, depending only on $z$ such that $H_{k}(x+z)-H_{k}(x) \leqq B(z)$ for all $x$ and all $k, k=0,1,2, \cdots$.

The condition imposed on $\left\{X_{n}\right\}$ in order to get this uniform bound is one which is also required in another connection in the proof of the renewal theorem in 3 . Hence asking that (2.1) be satisfied is not causing our main result to be burdened by an excess of hypotheses. 
LEMma 2-A. Let $\left\{X_{n}\right\}$ be a sequence of independent, non-negative random variables. If there exists a positive constant, $\alpha$, such that $\left(S_{n+k}-S_{n}\right) / k$ converges in probability to $\alpha$ as $k \rightarrow \infty$ uniformly in $n$, then (2.1) is satisfied. Furthermore,

(2.2) for any $\nu>0$ there exists $z^{\prime}>0$ depending only on $\nu$ such that for all $z \geqq z^{\prime}$ and for all $x, H(x+z)-H(x) \leqq z(\nu+1 / \alpha)$.

Proof. We first establish that $H_{k}(z)$ is finite for each $z$. For any $0<\rho<\alpha / 2$ there exists $k^{\prime}>0, k^{\prime}=k^{\prime}(\rho)$ such that for all $k \geqq k^{\prime}$ and for all $n$, $P\left(\left|\left(S_{n+k}-S_{n}\right) / k-\alpha\right| \geqq \rho\right) \leqq \rho$. Fix $z$ and choose $k \geqq \max \left(k^{\prime}, z /(\alpha-\rho)\right)$. Then for all $n$

$$
\left(\underset{j=n+1}{*} P\left(X_{j}<z\right)\right) \leqq\left(\underset{j=n+1}{*} P\left(X_{j}<k(\alpha-\rho)\right)\right) \leqq \rho .
$$

Therefore

$$
\begin{aligned}
H_{n}(z) \leqq & k+k\left(\underset{j=n+1}{*} P\left(X_{j}<k(\alpha-\rho)\right)\right)+k\left(\underset{j=n+1}{*+2 k} P\left(X_{j}<k(\alpha-\rho)\right)\right) \\
& +k\left(\underset{j=n+1}{*} P\left(X_{j}<k(\alpha-\rho)\right)\right)+\cdots \leqq k \sum_{\nu=0}^{\infty} \rho^{\nu}=k /(1-\rho)
\end{aligned}
$$

Since $\rho$ may be taken as small as we like, we not only have that $H_{n}(z)$ is finite for each $z$, but in addition we have found a bound which is uniform in $n$.

Next let

$$
J_{v}(x, z)=\max \left\{n: S_{v+n+N_{v}(x)+1}-S_{v+N_{v}(x)+1} \leqq z\right\} .
$$

When $v=0$ write $J_{0}(x, z)=J(x, z)$. Then for each $v, v=0,1,2, \cdots N_{v}(x+z)$ $\leqq 1+N_{v}(x)+J_{v}(x, z)$. Transposing and integrating yields

$$
\begin{aligned}
H_{v}(x+z)-H_{v}(x) & =\int\left(N_{v}(x+z)-N_{v}(\dot{x})\right) d P \\
& \leqq 1+\sum_{j=v}^{\infty} P\left(S_{j}-S_{v} \leqq x<S_{j+1}-S_{v}\right) H_{v+j+1}(z) .
\end{aligned}
$$

Fix $k \geqq \max \left(k^{\prime}, z /(\alpha-\rho)\right)$. Then

$$
\begin{aligned}
& H_{v}(x+z)-H_{v}(x) \\
& \quad \leqq 1+\sum_{j=v}^{\infty} P\left(S_{j}-S_{v} \leqq x<S_{j+1}-S_{v}\right) k /(1-\rho) \leqq 1+k(1-\rho) .
\end{aligned}
$$

$k$ depends on $z$ but is independent of $x$ and hence we have (2.1).

Next let $\nu>0$ be given. To get (2.2) we take $\rho$ so small that $1 /(\alpha-\rho)(1-\rho)$ $\leqq \nu / 2+1 / \alpha$. For this $\rho$ we pick $k^{\prime}$ as in the proof of (2.1). Let $z^{\prime \prime}=k^{\prime}(\alpha-\rho)$. For any $z \geqq z^{\prime \prime}$ choose $k$ so that $k(\alpha-\rho)<z \leqq(k+1)(\alpha-\rho)$. Then $k+1$ $\geqq \max \left(k^{\prime}, z /(\alpha-\rho)\right)$ and hence 


$$
\begin{aligned}
H(x+z)-H(x) & \leqq k /(1-\rho)+1+1 /(1-\rho) \\
& \leqq \frac{z k}{k(\alpha-\rho)(1-\rho)}+1+1 /(1-\rho) \\
& \leqq z[(\nu / 2+1 / \alpha)+1 / z+1 / z(1-\rho)] .
\end{aligned}
$$

If we let $z^{\prime}=\max \left(z^{\prime \prime}, 4 / \nu, 4 / \nu(\alpha-\rho)\right),(2.2)$ is proven.

In a continuous renewal sequence the statement analogous to

$$
\lim _{n \rightarrow \infty}\left(u_{n}-u_{n-1}\right)=0
$$

is:

(2.3) For any $h>0 \lim _{x \rightarrow \infty}[H(x+h)-H(x)-(H(x)-H(x-h))]=0$. However in the proof of Theorem 3 we will not require (2.3), but instead we will ask that the following condition be satisfied for some $n$.

(2.4-n) For any function $g$ on $(-\infty, \infty)$ with compact support and with $n+3$ continuous derivatives, and for any $h>0, \lim _{x \rightarrow \infty} g *[H(x+h)-H(x)]=0$.

Theorem 2. Let $\left\{X_{n}\right\}$ be a sequence of independent, non-negative random variables. If there exists a subsequence $\left\{X_{n_{k}}\right\}$ of $\left\{X_{n}\right\}$, a sequence $\left\{a_{k}\right\}$ of real numbers, and positive constants $M$ and $\omega$ such that

(A) for all $k, P\left(\left|X_{n_{k}}-a_{k}\right|<M\right) \geqq \omega$;

(B) if $\rho_{k}(t)$ is defined as in Theorem 1 , then for any $d>0$ there exists $u>0$, $u=u(d)$, such that $\sup _{k \geqq 1}\left|\rho_{k}(t)\right| \leqq 1-u$ for all $t$ satisfying $|t| \geqq d$;

(C) (2.1) is satisfied;

then (2.4-0) holds.

Proof. Since for fixed $x, \lim _{N \rightarrow \infty} \sum_{n=1}^{N}\left(*_{j=1}^{n} P\left(X_{j}<t\right)\right)=H(t)$ uniformly for all $t$ in the interval $[0, x]$, we can write

$$
\begin{aligned}
g *[H(x+h)-H(x)]= & \sum_{j=1}^{q} g * P\left(x \leqq S_{j}<x+h\right) \\
& +\int_{0}^{x+h} g *\left[H_{q}(x+h-t)-H_{q}(x-t)\right] d P\left(S_{q}<t\right) .
\end{aligned}
$$

As was the case in Theorem 1, our problem reduces to that of showing that

$$
\int_{0}^{x+h} g *\left[H_{q}(x+h-t)-H_{q}(x-t)\right] d P\left(S_{q}<t\right)
$$

approaches 0 as $q$ approaches $\infty$ uniformly in $x$.

Case 1. We first assume that for all $k, P\left(\left|X_{n k}-a_{k}\right|<M\right)=1$. Let $q^{\prime}$ $=\max \left\{n_{k}: n_{k} \leqq q\right\}$ and $U_{q}(x)=\left[*_{j=1 ; j \notin\left\{n_{k}\right\}}^{q} P\left(X_{j}<x\right)\right]$.

We have condition (2.1) and a uniform bound on $\left\{\left|X_{n_{k}}-a_{k}\right|\right\}$ so that for any $\nu>0$ there exists $Q^{\prime}=Q^{\prime}(\nu)$ such that for all $q \geqq Q^{\prime}$, 


$$
\begin{aligned}
& |(2.5)|=\left|\int_{0}^{x+h} g * U_{q} *\left[H_{q}(x+h-t)-H_{q}(x-t)\right] d P\left(\sum_{k=1}^{q^{\prime}} X_{n_{k}}<t\right)\right| \\
& \leqq 2 \nu+\left|\int_{\left|t-E\left(\sum_{k=1}^{q^{\prime}} X_{n_{k}}\right)\right| \leqq u q^{\prime}} g * U_{q} *\left[H_{q}(x+h-t)-H_{q}(x-t)\right] d P\left(\sum_{k=1}^{q^{\prime}} X_{n_{k}}<t\right)\right| .
\end{aligned}
$$

Next we let $W_{q^{\prime}}=\sum_{k=1}^{q^{\prime}} X_{n_{k}}$ and define a function $A_{q}(y)$ as follows:

$$
A_{q}(y)= \begin{cases}1 & \text { if }\left|y-E\left(W_{q^{\prime}}\right)\right| \leqq q^{\prime}, \\ \frac{1}{2}\left[\cos \left(y-E\left(W_{q^{\prime}}\right)+\nu q^{\prime}\right)+1\right] & \text { if }\left|y-\left(E\left(W_{q^{\prime}}\right)-\nu q^{\prime}-\pi / 2\right)\right|<\pi / 2, \\ \frac{1}{2}\left[\cos \left(y-E\left(W_{q^{\prime}}\right)-\nu q^{\prime}\right)+1\right] & \text { if }\left|y-\left(E\left(W_{q^{\prime}}\right)+\nu q^{\prime}+\pi / 2\right)\right|<\pi / 2, \\ 0 & \text { if }\left|y-E\left(W_{q^{\prime}}\right)\right| \geqq \pi+\nu q^{\prime} .\end{cases}
$$

If we write

$$
\int A_{q}(y) g * U_{q} *\left[H_{q}(x+h-y)-H_{q}(x-y)\right] d P\left(W_{q^{\prime}}<y\right)
$$

then we have that the absolute value of (2.5) is less than or equal to the absolute value of (2.6) plus $2 v$ for all $q \geqq Q^{\prime}$. We observe that

$$
A_{q}(y) q * U_{q} *\left[H_{q}(x+h-y)-H_{q}(x-y)\right]
$$

as a function of $y$ is continuous, is of bounded variation on $(-\infty, \infty)$, and is in $\mathscr{L}_{1}(-\infty, \infty)$ for each fixed $x$, and we apply Parseval's identity to get that

$$
|(2.6)|=\left|\iint e^{-i t y} A_{q}(y) g * U_{q} *\left[H_{q}(x+h-y)-H_{q}(x-y)\right] d y \prod_{k=1}^{q^{\prime}} \rho_{k}(t) d t\right| \text {. }
$$

The subsequence $\left\{X_{n_{k}}\right\}$ satisfies the hypotheses of Lemma 1-A so that there must exist positive constants $d$ and $b$ such that for all $k$ and for all $t, 0 \leqq|t|$ $\leqq d,\left|\rho_{k}(t)\right| \leqq 1-b t^{2}$. Let $R$ be such that the support of $g$ is contained in the interval $[-R, R]$ and let $C$ be such that for all $y,|g(y)|,\left|g^{\prime}(y)\right|,\left|g^{\prime \prime}(y)\right|$, and $\left|g^{\prime \prime \prime}(y)\right|$ are bounded by $C$. Then

$$
\begin{aligned}
& \left|\int_{-d}^{d} \int^{-i t y} A_{q}(y) q * U_{q} *\left[H_{q}(x+h-y)-H_{q}(x-y)\right] d y \prod_{k=1}^{q^{\prime}} \rho_{k}(t) d t\right| \\
& \leqq \int_{-d}^{d}\left|\int_{E\left(W_{q^{\prime}}\right)-\nu q^{\prime}}^{E\left(W_{q^{\prime}}\right)+\nu q^{\prime}-h}\left(e^{i t h}-1\right) e^{-i t y} g * U_{q} * H_{q}(x-y) d y\right|\left(1-b t^{2}\right)^{q^{\prime}} d t \\
& \quad+4 d C(2 \pi+h) B(2 R) \\
& \leqq 2 \int_{0}^{d} C B(2 R) t h \nu q^{\prime}\left(1-b t^{2}\right)^{q^{\prime}} d t+4 d C B(2 R)(h+2 \pi) \\
& \leqq(C / b) h B(2 R) \nu+4 d C(2 \pi+h) B(2 R) .
\end{aligned}
$$


Here $B(z)$ is the bound of $(2.1)$. We fix $d$ so small that $4 d C(2 \pi+h) B(2 H)<\nu$. Note that the estimate for the integral from $-d$ to $d$ is independent of both $x$ and $q$.

For all $|t| \geqq d$ integration by parts twice yields

$$
\left|\int e^{-i t y} A_{q}(y) g * U_{q} *\left[H_{q}(x+h-y)-H_{q}(x-y)\right] d y\right| \leqq \frac{2 C B(2 R)\left(\nu q^{\prime}+6 \pi\right)}{t^{2}} \text {. }
$$

Condition (B) of this theorem allows us to find $u, 0<u<1$, such that we can write

$$
\begin{gathered}
\int_{|t| \geq d}\left|\int e^{-i t y} A_{q}(y) g * U_{q} *\left[H_{q}(x+h-y)-H_{q}(x-y)\right] d y\right| \prod_{k=1}^{q^{\prime}}\left|\rho_{k}(t)\right| d t \\
\leqq \\
4\left(t^{\prime}-d\right) C B(2 R)\left(\nu q^{\prime}+2 \pi\right)(1-u)^{q^{\prime}} \\
+2 \int_{t^{\prime}}^{\infty} \frac{2 C B(2 R)\left(\nu q^{\prime}+6 \pi\right)(1-u)^{q^{\prime}} d t}{t^{2}} .
\end{gathered}
$$

For fixed $u,(1-u)^{q^{\prime}}\left(\nu q^{\prime}+6 \pi\right)$ is uniformly bounded for $q^{\prime}=0,1,2, \cdots$. Hence we may take $t^{\prime}$ so large that $\int_{t^{\circ}} 2 C B(2 R)\left(\nu q^{\prime}+6 \pi\right)(1-u)^{q^{\prime}} d t / t^{2} \leqq \nu$ for all $q^{\prime}$. Fix $t^{\prime}$ and choose $Q^{\prime \prime}$ so that for all $q \geqq Q^{\prime \prime}, 4 t^{\prime} C B(2 R)\left(\nu q^{\prime}+2 \pi\right)$ . $(1-u)^{q^{\prime}} \leqq \nu$. Therefore for all $q \geqq \max \left(Q^{\prime}, Q^{\prime \prime}\right)$,

$$
\left|\int_{0}^{x+h} g * U_{q} *\left[H_{q}(x+h-y)-H_{q}(x-y)\right] d P\left(W_{q^{\prime}}<y\right)\right| \leqq 6 \nu+\nu h C B(2 R) / b,
$$

$\nu$ is arbitrary and hence the proof for Case 1 is complete.

Case 2 . The case where $\omega$ must be taken less than 1 is handled by writing

$$
\begin{aligned}
& \left|\int_{0}^{x+h} g * U_{q} *\left[H_{q}(x+h-y)-H_{q}(x-y)\right] d P\left(W_{q^{\prime}}<y\right)\right| \\
& =\mid \int_{0}^{x+h} g * U_{q} *\left[H_{q}(x+h-y)\right. \\
& \left.-H_{q}(x-y)\right] d\left[\begin{array}{l}
q^{\prime} \\
\left.\underset{k=1}{*}\left(\nu_{k} L_{k}(y)+\left[1-\omega_{k}\right] K_{k}(y)\right)\right] \mid
\end{array}\right. \\
& \nu_{k}=P\left(\left|X_{n_{k}}-a_{k}\right|<M\right) \geqq \omega, \quad L_{k}(y)=P\left(X_{n_{k}}<y|| X_{n_{k}}-a_{k} \mid<M\right),
\end{aligned}
$$

and

$$
K_{k}(y)=\left(P\left(X_{n_{k}}<y\right)-\nu_{k} L_{k}(y)\right) /\left(1-\nu_{k}\right) .
$$

The proof is completed by using the binomial expansion and estimating as in Lemma 1-B.

Corollary 2-A. Let $\left\{X_{n}\right\}$ be a sequence of independent, non-negative random variables. If there exists a subsequence $\left\{X_{n_{k}}\right\}$ of $\left\{X_{n}\right\}$, a sequence of intervals $\left\{\left(b_{k}, c_{k}\right]\right\}$, and positive constants $M, R$, and $\omega$ such that 
(i) for all $k, c_{k}-b_{k}=2 M$;

(ii) for all $k, P\left(X_{n_{k}}<x\right)$ is absolutely continuous on $\left(b_{k}, c_{k}\right]$. Also for each $x \in\left(b_{k}, c_{k}\right], d P\left(X_{n_{k}}<x\right) / d x=f_{k}(x) \leqq R$ uniformly in $k$;

(iii) for all $k, P\left(b_{k}<X_{n_{k}}<c_{k}\right) \geqq \omega$;

(iv) (2.1) is satisfied;

then (2.4-0) holds.

Proof. Let $a_{k}=\left(b_{k}+c_{k}\right) / 2$. Then conditions (i) and (iii) immediately establish condition (A) of Theorem 2. (iv) is identical with (C) so that it remains only to show that condition (B) of Theorem 2 is satisfied. Assume that there exists some $d>0$, some sequence of real numbers $\left\{t_{j}\right\}$ with $\left|t_{j}\right| \geqq d$ for all $j$, and some subsequence $\left\{k_{j}\right\}$ of positive integers such that $\lim _{j \rightarrow \infty}\left|\rho_{k_{j}}\left(t_{j}\right)\right|=1$ where $\rho_{k}(t)=\int_{b_{k}}^{c_{k}}\left(1 / \nu_{k}\right) e^{i t x} f_{k}(x) d x$ and $\nu_{k}=P\left(b_{k}<X_{n_{k}}<c_{k}\right)$. We can assume that all the $t_{j}$ are positive. The unit circle is compact so that we can also assume that there exists a positive number $r$ such that for the subsequences $\left\{t_{j}\right\}$ and $\left\{k_{j}\right\}$,

$$
\lim _{j \rightarrow \infty} \int_{b_{k_{j}}}^{c_{k_{j}}}\left(1 / \nu_{k_{j}}\right)\left(1-\cos \left(r+x t_{j}\right)\right) f_{k_{j}}(x) d x=0 .
$$

Let $s>0$ be such that $1 / s \pi+2 / s M d<1 / 2$ and let

$$
E_{j}=\bigcup_{m=-\infty}^{\infty}\left[\frac{2 m \pi-r}{t_{j}}-\frac{\omega}{2 s M R t_{j}}, \frac{2 m \pi-r}{t_{j}}+\frac{\omega}{2 s M R t_{j}}\right] .
$$

On the complement of $E_{j}$ the function, $1-\cos \left(r+x t_{j}\right)$, is bounded away from 0 so that it must be the case that

$$
\lim _{j \rightarrow \infty} \int_{\left.\mid b_{k, j}, c_{k j}\right]-E_{j}} f_{k j}(x) d x=0 .
$$

At the same time

$$
\int_{\left[b_{k_{j}}, c_{j}\right] \cap E_{j}}\left(1 / \nu_{k_{j}}\right) f_{k_{j}}(x) d x \leqq \frac{R \omega}{\omega S M R t_{j}}\left(2+M t_{j} / \pi\right) \leqq 1 / 2 .
$$

But for all $j,\left(1 / \nu_{k_{j}}\right) \int_{b_{k}}^{c_{k}} f_{k_{j}}(x) d x=1$ and hence the corollary is proven.

REMARK. From the hypotheses of Corollary 2-A and one additional assumption it is actually possible to prove (2.3). The function $g$ in the statement of (2.4-0) can be replaced by the indicator function of the interval $(0, h]$. The smoothness properties of $g$ were needed only in estimating the tails of a certain integral. Because the functions, $f_{k}(x) / \nu_{k}$, are uniformly bounded, the functions, $\rho_{k}(t)$, are in $\mathscr{L}_{2}(-\infty, \infty)$. In addition to the assumptions of Corollary 2-A, if we ask that there exist positive constants $C$ and $t^{\prime}$ such that for all $k$ and all $t,|t| \geqq t^{\prime},\left|\rho_{k}(t)\right| \leqq C / \sqrt{ }(t)$, then the smoothness of $g$ is not required and (2.3) can be concluded.

In some special instances it is possible to establish (2.4-n) for some $n>0$ 
as a consequence of the presence of a subsequence, $\left\{X_{n_{k}}\right\}$, of random variables even though for each $k$,

$$
\limsup _{t \rightarrow \infty}\left|E\left(\exp i t X_{n_{k}}\right)\right|=1 \text {. }
$$

Lemma 2-B. Let $p_{1}, p_{2}, p_{3}, a, b$, and $c$ be real numbers with the following properties:

(i) each $p_{k}>0$ and $p_{1}+p_{2}+p_{3}=1$.

(ii) for no real numbers $m$ and $d$ and integers $k, k^{\prime}$, and $k^{\prime \prime}$ is it possible to write simultaneously $a=m+k d, b=m+k^{\prime} d, c=m+k^{\prime \prime} d$;

(iii) $(a-b) /(a-c)$ is an algebraic number;

then there exists some positive integer $n$ and real number $t^{\prime}$ such that for all $|t| \geqq t^{\prime}$,

$$
\left|p_{1} e^{i t a}+p_{2} e^{i t b}+p_{3} e^{i t c}\right| \leqq 1-1 / t^{n} .
$$

Proof. Let $r=\min \left(p_{1}, p_{2}, p_{3}\right)$ and $\rho(t)=\left(p_{1} e^{i t a}+p_{2} e^{i t b}+p_{3} e^{i t c}\right)$.

$$
\begin{aligned}
|\rho(t)|^{2}= & p_{1}^{2}+p_{2}^{2}+p_{3}^{2}+2 p_{1} p_{2} \cos t(a-b)+2 p_{1} p_{3} \cos t(a-c) \\
& +2 p_{2} p_{3} \cos t(b-c) \\
\leqq & p_{1}^{2}+p_{2}^{2}+p_{3}^{2}+2 p_{1} p_{2} \cos t(a-b)+2 p_{1} p_{3} \cos t(a-c)+2 p_{2} p_{3} .
\end{aligned}
$$

Denote by $k(t, a-b)$ the closest integer to $t(a-b) / 2 \pi$ and let $u=u(t, a-b)$ $=t(a-b)-2 \pi k(t, a-b)$. In the same manner define $m(t, a-c)$ and $v$ $=v(t, a-c)$. Let $E=\{t:|u| \geqq \pi / 2\} \cup\{t:|v| \geqq \pi / 2\}$. For $t$ in $E$,

$$
\begin{aligned}
|\rho(t)|^{2} & \leqq p_{1}^{2}+p_{2}^{2}+p_{3}^{2}+2 p_{1} p_{2}+2 p_{1} p_{3}+2 p_{2} p_{3}-2 r^{2} \\
& \leqq 1-2 r^{2} \leqq 1-2 r^{2}+r^{4} .
\end{aligned}
$$

Therefore for $t$ in $E, \rho(t) \leqq 1-r^{2}$, and hence we need only concern ourselves with the complement of $E$. If both $|u|<\pi / 2$ and $|v|<\pi / 2$ then

$$
\begin{aligned}
|\rho(t)|^{2} & \leqq p_{1}^{2}+p_{2}^{2}+p_{3}^{2}+2 p_{1} p_{2}\left(1-[2 u / \pi]^{2}\right)+2 p_{1} p_{3}\left(1-[2 v / \pi]^{2}\right)+2 p_{2} p_{3} \\
& \leqq 1-2 r^{2}\left([2 u / \pi]^{2}+[2 v / \pi]^{2}\right) \leqq 1-r^{2}(|2 u / \pi|+|2 v / \pi|)^{2} .
\end{aligned}
$$

We can assume that $|a-b|<|a-c|$ so that for all $t,|k(t, a-b)|=|k|$ $\leqq|m(t, a-c)|=m$.

$$
\begin{aligned}
|\rho(t)|^{2} & \leqq 1-\frac{r^{2}|2 \pi m+v|^{2}}{4}\left(\frac{m u}{m(2 \pi m+v)}-\frac{k v}{m(2 \pi m+v)}\right) \\
& \leqq 1-\frac{r^{2}|t(a-c)|^{2}}{4}\left|\frac{2 \pi k+u}{2 \pi m+v}-\frac{k}{m}\right|^{2}=1-\frac{r^{2}|t(a-c)|^{2}}{4}\left|\frac{a-b}{a-c}-\frac{k}{m}\right|^{2} .
\end{aligned}
$$

If the degree of the algebraic number $(a-b) /(a-c)$ is $j \geqq 2$ then there must exist a constant $w>0$ such that for all integers, $k$ and $m$,

$$
\left|\frac{a-b}{a-c}-\frac{k}{m}\right|>\frac{w}{m^{v}} \text {. }
$$


Here $j$ must be greater than 1 for if $(a-b) /(a-c)$ is rational then $a-b$, $a-c$, and $b-c$ must be integral multiples of the same real number $x$. In this case $|\rho(2 \pi / x)|=1$, thus violating condition (ii) of the lemma. Finally

$$
|\rho(t)|^{2} \leqq 1-r^{2}|t(a-c)|^{2} w^{2} / 4 m^{2 j} \text {. }
$$

For large $|t|, 1+|v| /|t(a-c)| \leqq 2$ so that there exists some $t^{\prime}>0$ such that for all $|t| \geqq t^{\prime}$,

$$
\begin{aligned}
\left|\rho_{k}(t)\right|^{2} & \leqq 1-\frac{r^{2} w^{2}|t(a-c)|^{2}(2 \pi)^{2 j}}{4(t(a-c)-v)^{2 j}} \\
& \leqq 1-\frac{r^{2} w^{2}|a-c|^{2-2 j}(2 \pi)^{2 j}}{\left(t^{2 j-2}\right)\left(2^{2 j+2}\right)} \\
& \leqq 1-2 / t^{2 j-1} \leqq 1-2 / t^{2 j-1}+1 / t^{4 j-2}
\end{aligned}
$$

Therefore for all $|t| \geqq t^{\prime},|\rho(t)| \leqq 1-1 / t^{n}$ where $n=2 j-1$.

Let $E=\left\{x:|x-p / q|<1 / q^{3}\right\}$ has infinitely many solutions in integers $p$ and $q$. Then the Lebesgue measure of the set $E$ is 0 . This fact is stated as 198 on p. 168 of Hardy and Wright [9]. Therefore for each $x$ not in $E$ there exists an integer $q^{\prime}=q^{\prime}(x)$ such that for all integers $p$ and $q, q \geqq q^{\prime}$, $|x-p / q|>1 / q^{3}$.

Lemma 2-C. Let $p_{1}, p_{2}, p_{3}, a, b$, and $c$ be real numbers satisfying conditions (i) and (ii) of Lemma 2-B. In addition if $(a-b) /(a-c)$ is not in $E$ then there exists a real number $t^{\prime}$ such that for all $|t| \geqq t^{\prime}$,

$$
\left|p_{1} e^{i t a}+p_{2} e^{i t b}+p_{3} e^{i t c}\right| \leqq 1-1 / t^{n}
$$

where $n=2 \cdot 3-1=5$.

Proof. The proof is the same as in Lemma 2-B with the integer 3 playing the role of the algebraic order $j$. The inequality of the preceding paragraph replaces the inequality of Lemma $2-B$ which deals with approximations of algebraic numbers by rationals.

Lemmas 2-B and 2-C can be used to draw from Theorem 2 another corollary.

CoRollary 2-B. Let $\left\{X_{n}\right\}$ be a sequence of non-negative independent random variables. If there exists a subsequence, $\left\{X_{n_{k}}\right\}$, of $\left\{X_{n}\right\}$, a sequence $\left\{a_{k}\right\}$ of real numbers, positive constants $M, \omega$, and $t^{\prime}$, and a positive integer $n$ such that:

(i) for all $k, P\left(\left|X_{n_{k}}-a_{k}\right|<M\right) \geqq \omega$;

(ii) for any $d>0$ and $t^{\prime \prime} \geqq d$ there exists $u>0, u=u\left(d, t^{\prime \prime}\right)$ such that for all $t$ satisfying $d \leqq|t| \leqq t^{\prime \prime}, \sup _{k \geqq 1}\left|\rho_{k}(t)\right| \leqq 1-u$ where $\rho_{k}(t)$ is defined as in Theorem 2;

(iii) for all $k$ and all $|t| \geqq t^{\prime},\left|\rho_{k}(t)\right| \leqq 1-1 / t^{n}$;

(iv) (2.1) is satisfied;

then (2.4-n) holds. 
Proof. Let $g(x)$ have compact support on $(-\infty, \infty)$ and have $n+3$ continuous derivatives. In the proof of Theorem 2 the integral, (2.6), was transformed by Parseval's identity and estimated in three parts. Showing that

$$
\int_{|t| \geq t^{\prime \prime}}\left|\left\{\int e^{-i t y} A_{q}(y) g * U_{q} *\left[H_{q}(x+h-y)-H_{q}(x-y)\right] d y \prod_{k=1}^{q^{\prime}} \rho_{k}(t)\right\}\right| d t
$$

can be made small uniformly in $q^{\prime}$ and $x$ by taking $t^{\prime \prime}$ large is all that is required to complete this proof, for the estimation on the remaining part of the integral can be made as in Theorem 2. Taking $t^{\prime \prime} \geqq t^{\prime}$ and integrating by parts $n+2$ times gives

$$
\begin{aligned}
\int_{|t| \geqq t^{\prime \prime}} \mid & \int^{-i t y} A_{q}(y) g * U_{q} *\left[H_{q}(x+h-y)-H_{q}(x-y)\right] d y|| \prod_{k=1}^{q^{\prime}} \rho_{k}(t) \mid d t \\
\leqq & 2 \int_{t^{\prime \prime}}^{\infty} \int \mid\left(1 / t^{n+2}\right) e^{-i t y} A_{q}(y) \frac{d^{(n+2)}}{d y^{(n+2)}}\left\{g * U _ { q } * \left[H_{q}(x+h-y)\right.\right. \\
& \left.\left.-H_{q}(x-y)\right]\right\} \mid d y\left(1-1 / t^{n}\right)^{q^{\prime}} d t \\
& +\sum_{k=1}^{n+2} 2 \int_{t^{\prime}}^{\infty} \int_{E_{q}}\left|\left(1 / t^{k}\right) e^{-i t y} \frac{d}{d y}\left(A_{q}(y)\right)\right| \\
& \left.\left.\cdot \sqrt{\frac{d^{(k-1)}}{d y^{(k-1)}}\{g *} U_{q} * \mid H_{q}(x+h-y)-H_{q}(x-y)\right]\right\} \mid d y d t
\end{aligned}
$$

where

$$
\begin{aligned}
E_{q} & =\left\{y:\left|y-\left[E\left(W_{q^{\prime}}\right)-\nu q^{\prime}-\pi / 2\right]\right| \leqq \pi / 2\right\} \\
& \cup\left\{y:\left|y-\left[E\left(W_{q^{\prime}}\right)+\nu q^{\prime}+\pi / 2\right]\right| \leqq \pi / 2\right\} .
\end{aligned}
$$

The first term on the right-hand side of the above inequality is less than or equal to $\int_{t^{\prime \prime}}^{\infty}\left(1 / t^{n+2}\right)\left(1-1 / t^{n}\right)^{q^{\prime}} C_{q^{\prime}} d t \leqq 2 C / t^{\prime \prime}$ where $C$ is a constant depending on $h, n$, the length of an interval containing the support of $g(x)$, and on the derivatives of $g(x)$, but not on $q^{\prime}$. The Lebesgue measure of $E_{q}$ is less than or equal to $2 \pi$ for all $q . n$ is fixed and we have (2.1) so that we can make the sum on the right as small as we wish uniformly in $q^{\prime}$ and $x$ by taking $t^{\prime \prime}$ large. Therefore for any $\nu>0$ there exists $t^{\prime \prime}>0$ depending only on $\nu$ such that for all $q$ and all $x$

$$
\int_{|t| l t^{\prime \prime}}\left|\int e^{-i t y} A_{q}(y) g * U_{q} *\left[H_{q}(x+h-y)-H_{q}(x-y)\right] d y \prod_{k=1}^{q^{\prime}}\right| \rho_{k}(t)|| d t \leqq \nu .
$$

This completes the proof.

REMARK. It should be pointed out that the constant, $w$, in Lemma 2-B depends on the magnitude of the coefficients and the degree of the poly- 
nomial of smallest degree for which $(a-b) /(a-c)$ is a root. If for each random variable in the subsequence $\left\{X_{n_{k}}\right\}$ of Corollary 2-B there corresponds a constant, $w_{k}$, and if there are infinitely many distinct elements in $\left\{w_{k}\right\}$, then it is possible that $\inf _{k}\left\{w_{k}\right\}=0$ in which case it might not be possible to find the $t^{\prime}$ of Corollary 2-B. On the positive side example 4.3 shows how Corollary 2-B can be used to derive a mildly interesting result.

A result like that of Corollary 1-C can also be deduced from Theorem 2.

Corollary 2-C. Let $\left\{X_{n}\right\}$ be a sequence of non-negative independent random variables. If there exists a subsequence, $\left\{X_{n_{k}}\right\}$, a sequence of real numbers $\left\{a_{k}\right\}$, and positive constants $M, \omega, d$, and $m$ such that:

(i) for all $k, P\left(\left|X_{n k}-a_{k}\right|<M\right) \geqq \omega$;

(ii) for any $b>0$ there exists $u>0, u=u(b)$, such that for all $t$ satisfying $b \leqq|t| \leqq \pi / d$, $\sup _{k \geqq 1}\left|\rho_{k}(t)\right| \leqq 1-u$ where $\rho_{k}(t)$ is defined as in Theorem 2;

(iii) for all $k, \sum_{j=0}^{\infty} P\left(x_{n_{k}}=m+j d\right)=1$;

(iv) (2.1) is satisfied;

then for any $h>0, \lim _{x \rightarrow \infty}[(H(x)-H(x-h))-(H(x-d)-H(x-d-h))]=0$.

Proof. In the notation of Theorem 2, we need only show that

$\lim _{q \rightarrow \infty}\left|\int_{0}^{x} g *\left[H_{q}(x-y)-H_{q}(x-y-h)\right] d\left[P\left(W_{q^{\prime}}<y\right)-P\left(W_{q^{\prime}}<y-d\right)\right]\right|=0$

uniformly in $x$. But this expression is less than or equal to

$$
\begin{aligned}
& B(h) \int\left|d\left[P\left(W_{q^{\prime}}<y\right)=P\left(W_{q^{\prime}}<y-d\right)\right]\right| \\
& \quad=B(h) \sum_{j}\left|P\left(\sum_{k=1}^{q^{\prime}} X_{n_{k}}=m q^{\prime}+j d\right)-P\left(\sum_{k=1}^{q^{\prime}} X_{n_{k}}=m q^{\prime}+(j-1) d\right)\right|
\end{aligned}
$$

where $B(h)$ is the bound of $(2.1)$. The techniques of $\$ 1$ applied to the sum on $j$ yield the desired result.

This corollary can be combined with Corollary 3-A to give a further renewal theory result.

For further conditions which imply (2.3) or, for some $n,(2.4-n)$ the reader is referred to Theorem 4 in a paper by W. L. Smith [12]. Smith has limited success in handling random variables whose characteristic functions, while never unity for $T \neq 0$, have moduli equal to 1 for some $T \neq 0$. The methods of this section are of no use in treating such random variables. He is also able to say something about nonlattice probability distributions which do not satisfy the conditions of Corollary 2-B.

3. A renewal theorem. In this section conditions will be found to insure that there exists some positive constant, $\alpha$, for which 


$$
\lim _{z \rightarrow \infty} \frac{H(x+z)-H(x)}{z}=\frac{1}{\alpha}
$$

uniformly in $x$. By assuming in addition that either (2.3) is satisfied or that for some $n, n \geqq 0,(2.4-n)$ holds, we are able to conclude that

$$
\text { for any } h>0, \quad \lim _{x \rightarrow \infty}[H(x)-H(x-h)]=\frac{h}{\alpha} \text {. }
$$

If the random variables are integer valued, then the additional assumption is that $\lim _{n \rightarrow \infty}\left[u_{n}-u_{n-1}\right]=0$ and the conclusion is

$$
\lim _{n \rightarrow \infty} u_{n}=1 / \alpha \text {. }
$$

The case when $\alpha=\infty$ is also discussed.

DEFINITION. Let $\left\{X_{n}\right\}$ be a sequence of random variables, the set $A(x, y)$ is defined by

$$
A(x, y)=\bigcap_{k=1}^{\infty}\left\{\left[\sum_{j=1}^{k} X_{j}<x\right] \cup\left[\sum_{j=1}^{k} X_{j} \geqq x+y\right]\right\} .
$$

Lemma 3-A. Let $\left\{X_{n}\right\}$ be a sequence of non-negative independent random variables with $S_{n}=\sum_{k=1}^{n} X_{k}$. If

(3.3) there exists a positive constant $\alpha$ so that $\left(S_{n+k}-S_{n}\right) / k$ converges in probability to $\alpha$ as $k$ approaches infinity uniformly in $n$, and if

$$
\lim _{y \rightarrow \infty} P(A(x, y))=0
$$

uniformly in $x$, then for any $\nu>0$ there exists $z^{\prime}>0$ depending only on $\nu$ such that for all $x$ and for all $z \geqq z^{\prime},(H(x+z)-H(x)) / z \geqq 1 / \alpha-\nu$.

Proof. First we show that for any $\nu>0$ there exists $z^{\prime \prime}>0, z^{\prime \prime}=z^{\prime \prime}(\nu)$ such that for all $j, j=0,1,2, \cdots$ and all $z \geqq z^{\prime \prime}, H_{j}(z) \geqq z(1 / \alpha-\nu)$. We have $H_{j}(z)=\sum_{n=j+1}^{\infty}\left(*{ }_{k=j+1}^{n} P\left(X_{k}<z\right)\right)$. For any $\rho>0$ there exists $N$ such that for all $n \geqq N$ and all $v, P\left(S_{v+n}-S_{v}<n(\alpha+\rho)\right) \geqq 1-\rho$. Let $z^{\prime \prime}$ $=(N-1)(\alpha+\nu)$. If $z>z^{\prime \prime}$ we can find $n(z)=n$ so that $(n-1)(\alpha+\nu)<z$ $\leqq n(\alpha+\nu)$ and so that $n \geqq N$. For this $z$ and $n$ we can write

$$
H_{j}(z) \geqq \sum_{i=j+1}^{n}\left(\underset{k=j+1}{*} P\left(X_{k}<z\right)\right) \geqq n(1-\rho)
$$

and hence $H_{j}(z) / z \geqq n(1-\rho) / z \geqq(1-\rho) /(\alpha+\rho)$. But $\rho$ is arbitrary so we have the first of the desired inequalities. Define $Q(x, z)$ by

$$
Q(x, z)=\max \left\{n: X_{N(x)+1}+X_{N(x)+2}+\cdots+X_{N(x)+n}<z\right\} .
$$

Then 


$$
\begin{aligned}
E(Q(x, z))= & \sum_{k=0}^{\infty} P(Q(x, z)>k) \\
= & \sum_{k=0}^{\infty} \sum_{j=0}^{\infty} P\left(S_{j}<x, S_{n+1} \geqq x, N_{j}(z)>k\right) \\
\geqq & \sum_{k=0}^{\infty} \sum_{j=0}^{\infty} P\left(x-z^{1 / 2} \leqq S_{j}<x \leqq S_{j+1}<x+z^{1 / 2}\right) \\
& \cdot P\left(N_{j+1}\left(z-2 z^{1 / 2}\right)>k\right) \\
= & \sum_{j=0}^{\infty} P\left(x-z^{1 / 2} \leqq S_{j}<x \leqq S_{j+1}<x+z^{1 / 2}\right) H_{j+1}\left(z-2 z^{1 / 2}\right) .
\end{aligned}
$$

Now $N(x+z) \geqq N(x)+Q(x, z)$ so that

$$
\begin{aligned}
& \frac{H(x+z)-H(x)}{z} \\
& \geqq \sum_{j=0}^{\infty} P\left(x-z^{1 / 2} \leqq S_{j}<x \leqq S_{j+1} \leqq x+z^{1 / 2}\right)\left[\frac{H_{j+1}\left(z-2 z^{1 / 2}\right)}{\left(z-2 z^{1 / 2}\right)}\right]\left[\frac{\left(z-2 z^{1 / 2}\right)}{z}\right] .
\end{aligned}
$$

But $\bigcup_{j=0}^{\infty}\left\{x-z^{1 / 2} \leqq S_{j}<x \leqq S_{j+1}<x+z^{1 / 2}\right\}$ contains the complement of the set $A\left(x-z^{1 / 2}, z^{1 / 2}\right) \cup A\left(x, z^{1 / 2}\right)$ and hence for any $\nu>0$ there exists $z^{\prime}>0$ such that for all $z \geqq z^{\prime}, \sum_{j=0}^{\infty} P\left(x-z^{1 / 2} \leqq S_{j}<x \leqq S_{j+1}<x+z^{1 / 2}\right)$ $\geqq 1-\nu$ uniformly in $x$. Also for all $z \geqq z^{\prime},\left[H_{j+1}\left(z-2 z^{1 / 2}\right) /\left(z-2 z^{1 / 2}\right)\right]$ $\geqq 1 / \alpha-\nu$ uniformly in $j$ and $2 z^{1 / 2} / z \leqq \nu$. Therefore for all $x$ and for all $z \geqq z^{\prime}$ we have

$$
\frac{H(x+z)-H(x)}{z} \geqq(1-\nu)(1 / \alpha-\nu)(1-\nu) .
$$

$\nu$ is arbitrary and thus we have the desired result.

The next three lemmas translate conditions (3.3) and (3.4) into more manageable terms.

Lемма 3-B. Let $\left\{X_{n}\right\}$ be a sequence of independent non-negative random variables. If there exist positive constants $\alpha$ and $N$ and a probability distribution function $G(x)$ such that:

(i) $\lim _{k \rightarrow \infty} 1 / k \sum_{j=1}^{k} E\left(X_{n+j}\right)=\alpha$ uniformly for all $n \geqq N$;

(ii) for all $x$ and for all $n \geqq N, P\left(X_{n}<x\right) \geqq G(x)$ and $\int x d G(x)<\infty$; then (3.3) and(3.4) hold.

Proof. We shall show first that condition (3.3) is satisfied. Set $S_{n}=$ $\sum_{k=1}^{n} X_{k}$. For any $\nu>0$ and $n<N$, 


$$
\begin{aligned}
& P\left(\left|\frac{S_{n+k}-S_{n}}{k}-\alpha\right| \geqq \nu\right) \\
& \leqq P\left(\frac{k(\alpha-\nu / 2)}{k-(N-n)} \leqq \frac{S_{n+k}-S_{N}}{k-(N-n)} \leqq \frac{k(\alpha+\nu / 2)}{k-(N-n)}\right) \\
& \quad+P\left(\left|\frac{S_{N}-S_{n}}{k}\right| \geqq \nu / 2\right) .
\end{aligned}
$$

$N$ is fixed so that there exists $k^{\prime}$ such that for all $k>k^{\prime}$ and all $n, 1 \leqq n<N$, $k(\alpha-\nu / 2) /(k-(N-n)) \geqq \alpha-\nu$ and $k(\alpha+\nu / 2) /(k-(N-n)) \leqq \alpha+\nu$. Also we can always find a $k^{\prime \prime}$ such that for all $k>k^{\prime \prime} P\left(\left|\left(S_{N}-S_{n}\right) / k\right| \geqq \nu / 2\right)$ $\leqq \nu$ for each $n, n \leqq N$. Therefore the problem of showing that conditions (i) and (ii) imply condition (3.3) reduces to that of showing that (i) and (ii) imply (3.3) under the additional restriction that $N=0$. To establish (3.3) we assume $N=0$ and proceed with a truncation argument.

For any $p>0$ define $X_{k}(p)=X_{k}$ if $X_{k}<p$ and $X_{k}(p)=0$ if $X_{k} \geqq p$. For any $\nu>0$

$$
\begin{aligned}
& P\left(\left|\frac{S_{n+p}-S_{n}}{p}-\alpha\right| \geqq \nu\right) \leqq P\left(\bigcup_{k=1}^{p}\left\{X_{n+k} \geqq p\right\}\right) \\
& \quad+P\left(\frac{1}{p}\left|\sum_{k=1}^{p}\left(X_{n+k}(p)\right)-E\left(X_{n+k}(p)\right)\right| \geqq \nu / 2\right) \\
& \quad+P\left(\left|\frac{1}{p}\left(\sum_{k=1}^{p} E\left(X_{n+k}(p)\right)\right)-\alpha\right| \geqq \nu / 2\right) \\
& \leqq p(1-G(p))+\frac{4}{(p \nu)^{2}} E\left(\sum_{k=1}^{p}\left(X_{n+k}^{2}(p)\right)\right) \\
& \quad+P\left(\left|\left(\frac{1}{p} \sum_{k=1}^{p} E\left(X_{n+k}\right)\right)-\alpha\right| \geqq \nu / 4\right) \\
& \quad+P\left(\frac{1}{p} \sum_{k=1}^{p} \int_{x \geqq p} x d P\left(X_{n+k}<x\right) \geqq \nu / 4\right) \\
& \leqq p(1-G(p))+8 \int_{0}^{p} \frac{x(1-G(x)) d x}{p \nu^{2}} \\
& \quad+P\left(\left|\left(\frac{1}{p} \sum_{k=1}^{p} E\left(X_{n+k}\right)\right)-\alpha\right| \geqq \nu / 4\right) \\
& \left.\quad+G(p))+\int_{p}^{\infty}(1-G(x)) d x \geqq \nu / 4\right) \\
& \quad+P\left(1-\frac{1}{p}\right)
\end{aligned}
$$


The fact that $\int x d G(x)<\infty$ guarantees that the first, second, and fourth terms on the right in the above inequality can be made small by taking $p$ large. Condition (i) implies that the third term is 0 for all sufficiently large $p$.

Condition (3.4) is more easily verified. We make use of the fact that we have (3.3) and hence the uniform bound, $B(z)$, of (2.1).

$$
\begin{gathered}
P(A(x, y)) \leqq \sum_{j=0}^{\infty} \sum_{k=0}^{\mid x+1]} P\left(x-k-1<S_{j} \leqq x-k\right) P\left(X_{j+1} \geqq y+k\right) \\
\leqq \sum_{j=0}^{N} \sum_{k=0}^{[x+1]} P\left(x-k-1<S_{j} \leqq x-k\right) \\
P\left(X_{j+1} \geqq(y+k)\right)+B(1) \sum_{k=0}^{\infty}(1-G(y+k)) \\
\leqq \\
\sum_{j=0}^{N} P\left(X_{j+1} \geqq y\right)+B(1) \int_{y-1}^{\infty}(1-G(x)) d x .
\end{gathered}
$$

But $N$ is fixed and $\int x d G(x)=\int(1-G(x)) d x<\infty$ so that the terms on the right can be made small by taking $y$ large. The estimates are independent of $x$ so (3.4) is satisfied.

It is possible to have (3.3) and (3.4) satisfied without condition (ii) of Lemma 3 -B being satisfied.

Lemмa 3-C. Let $\left\{X_{n}\right\}$ be a sequence of independent non-negative random variables. If there exist positive constants $\alpha$ and $N$ such that:

(i) $\lim _{k \rightarrow \infty}(1 / k) \sum_{j=1}^{k} E\left(X_{n+j}\right)=\alpha$ uniformly for all $n>N$;

(ii) $\lim _{b \rightarrow \infty} \int_{b}^{\infty} x d P\left(X_{n}<x\right)=0$ uniformly for all $n>N$;

(iii) there exist positive constants $C$ and $z$ such that for all $k, P\left(X_{k} \geqq z\right)$ $\leqq C / k$;

then (3.3) and (3.4) hold.

Proof. Let $G(x)=\inf _{n \geqq 1}\left\{P\left(X_{n}<x\right)\right\}$. We claim that $\lim _{x \rightarrow \infty} x(1-G(x))$ $=0$. Assume that there exists a sequence $\left\{x_{k}\right\}$ such that $\lim _{k \rightarrow \infty}\left(x_{k}\right)=\infty$ and such that for all $k, x_{k}\left(1-G\left(x_{k}\right)\right) \geqq 5 \nu$ where $\nu$ is some positive number. Choose $b^{\prime}$ so that if $b \geqq b^{\prime}$ then

$\nu \geqq-\int_{b}^{\infty} x d\left(1-P\left(X_{n}<x\right)\right)=b\left(1-P\left(X_{n}<b\right)\right)+\int_{b}^{\infty}\left(1-P\left(X_{n}<x\right)\right) d x$.

Hence for any $x_{k}>b^{\prime}$ we can write $\nu \geqq x_{k}\left(1-P\left(X_{n}<x_{k}\right)\right)$. But this estimate is uniform in $n$ so that for fixed $x_{k}>b^{\prime}$ we can find an $n$ such that $\nu \geqq x_{k}\left(1-P\left(X_{n}<x_{k}\right)\right) \geqq x_{k}\left[1-G\left(x_{k}\right)-\nu / x_{k}\right]$ or $2 \nu \geqq x_{k}\left(1-G\left(x_{k}\right)\right) \geqq 5 \nu$. Therefore it must be the case that $\lim _{x \rightarrow \infty} x(1-G(x))=0$.

Just as in Lemma $3-\mathrm{B}$ we need only consider the case $N=0$ when attempting to establish (3.3). Also from Lemma 3-B we can write 


$$
\begin{aligned}
& P\left(\left|\frac{S_{n+k}-S_{n}}{k}-\alpha\right| \geqq \nu\right) \\
& \leqq k(1-G(k))+\frac{4}{(k \nu)^{2}} E\left(\sum_{j=1}^{k}\left[X_{n+j}(k)-E\left(X_{n+j}(k)\right)\right]\right)^{2} \\
& \quad+P\left(\left|\frac{1}{k} \sum_{j=1}^{k} E\left(X_{n+j}\right)-\alpha\right| \geqq \nu / 4\right)+P\left(\frac{1}{k} \sum_{j=1}^{k} \int_{k}^{\infty} x d P\left(X_{n+j}<x\right) \geqq \nu / 4\right)
\end{aligned}
$$

where $\nu$ is positive and arbitrary. The fact that $\lim _{x \rightarrow \infty} x(1-G(x))=0$ implies that the first and second terms on the right approach 0 as $k$ approaches infinity uniformly in $n$. The third and fourth terms on the right are 0 for all large $k$ and all $n$ as a consequence of conditions (i) and (ii). Therefore condition (3.3) is satisfied.

Turning to condition (3.4) we estimate using (3.3). For any $0<\nu<\alpha / 2$ there exists $k^{\prime}>0$ such that for all $k \geqq k^{\prime}, P\left(\left|S_{k} / k-\alpha\right| \geqq \nu\right) \leqq \nu$. Then

$$
P(A(x, y)) \leqq 2 \nu+\sum_{j=\mid x /(\alpha+\nu)]}^{1+[x /(\alpha-\nu)]} \sum_{k=0}^{[x+1]} P\left(x-k-1<S_{j} \leqq x-k\right) P\left(X_{j+1} \geqq y+k\right)
$$

whenever $x \geqq\left(k^{\prime}+1\right)(\alpha+\nu)$. Taking $y>z, \quad x \geqq\left(k^{\prime}+1\right)(\alpha+\nu)$, and using (iii) gives

$$
P(A(x, y)) \leqq 2 \nu+\sum_{j=|x /(\alpha+\nu)|}^{1+|x /(\alpha-\nu)|} C /(j+1) \leqq 2 \nu+\frac{2 C\left(1+\nu x /\left(\alpha^{2}-\nu^{2}\right)\right)}{1+x /(\alpha+\nu)} .
$$

Let $x^{\prime}$ be such that for all $x \geqq x^{\prime}, 2 C\left(1+\nu x /\left(\alpha^{2}-\nu^{2}\right)\right) /(1+x /(\alpha+\nu))$ $\leqq 2 \nu C\left(1+4 \alpha^{2} / 3\right) /(1 / 2 \alpha)$. Set $x^{\prime \prime}=\max \left(\left(k^{\prime}+1\right)(\alpha+\nu), x^{\prime}\right)$. We can find $z^{\prime}>0$ such that for all $y>z^{\prime}$ and all $x \leqq x^{\prime \prime}$,

$$
\begin{aligned}
P(A(x, y)) & \leqq \nu+\sum_{j=0}^{1+\left|x^{\prime} /(\alpha-\nu)\right|} \sum_{k=0}^{|x+1|} P\left(x-k-1<S_{j} \leqq x-k\right) P\left(X_{j+1} \geqq y+k\right) \\
& \leqq \sum_{j=0}^{1+\left|x^{\prime} /(\alpha-\nu)\right|} P\left(X_{j+1} \geqq y\right)+\nu \leqq 2 \nu .
\end{aligned}
$$

Therefore for all $y>\max \left(z, z^{\prime}\right), P(A(x, y))$ is bounded by an expression which is independent of $x$ and which can be made arbitrarily small by choosing $\nu$ near 0 . This establishes (3.4).

It is also possible to have (3.3) and (3.4) satisfied when $\alpha$ is not the Cesaro average of a sequence of expected values.

Lемма 3-D. Let $\left\{X_{n}\right\}$ be a sequence of non-negative independent random variables. If there exist positive constants $\alpha$ and $M$ such that:

(i) $\lim _{n \rightarrow \infty}(1 / n) \sum_{j=1}^{n} \alpha(k+j)=\alpha$ uniformly in $k$ where 


$$
\alpha(k)=\int_{0}^{M} x d P\left(X_{k}<x\right) ;
$$

(ii) $\sum_{k=1}^{\infty} P\left(X_{k} \geqq M\right)<\infty$;

then (3.3) and (3.4) are satisfied.

Proof. Let $\nu>0$ be given. There exists $N$ such that $\sum_{j=n}^{\infty} P\left(X_{j} \geqq M\right) \leqq \nu$ for all $n \geqq N$. Taking $n \geqq N$ and using the truncation notation of Lemma 3 -B we get

$$
\begin{aligned}
P\left(\left|\frac{S_{n+k}-S_{n}}{k}-\alpha\right| \geqq \nu\right) \leqq & P\left(\bigcup_{j=n}^{\infty}\left\{X_{j} \geqq M\right\}\right) \\
& +P\left(\frac{1}{k}\left|\sum_{j=1}^{k}\left(X_{n+j}(M)\right)-\alpha(n+j)\right| \geqq \nu / 2\right) \\
& +P\left(\left|\frac{1}{k} \sum_{j=1}^{k} \alpha(n+j)-\alpha\right| \geqq \nu / \rho\right) \\
\leqq & +\frac{4 k M^{2}}{(\nu k)^{2}}+P\left(\left|\frac{1}{k} \sum_{j=1}^{k} \alpha(n+j)-\alpha\right| \geqq \nu / 2\right) .
\end{aligned}
$$

Each term can be made less than $\nu$ uniformly for all $n \geqq N$ by taking $k$ greater than some $k^{\prime}$. For $n<N$,

$$
\begin{aligned}
P\left(\left|\frac{S_{n+k}-S_{n}}{k}-\alpha\right| \geqq \nu\right) \\
\leqq P\left(\left|\frac{S_{n+k}-S_{N}}{k}-\alpha\right| \geqq \nu / \alpha\right)+P\left(\frac{S_{N}-S_{n}}{k} \geqq \nu / 2\right) \\
\leqq P\left(\frac{k(\alpha-\nu / 2)}{k-(N-n)} \leqq \frac{S_{n+k}-S_{N}}{k-(N-n)} \leqq \frac{k(\alpha+\nu / 2)}{k-(N-n)}\right) \\
\quad+P\left(S_{N} \geqq k \nu / 2\right) .
\end{aligned}
$$

$N$ is fixed and therefore there exists $k^{\prime \prime}$ such that whenever $k>k^{\prime \prime}$ and $n<N$,

$$
P\left(\left|\frac{\left(S_{n+k}-S_{n}\right)}{k}-\alpha\right| \geqq \nu\right) \leqq 4 \nu .
$$

If $k \geqq \max \left(k^{\prime}, k^{\prime \prime}\right)$ then $P\left(\left|\left(S_{n+k}-S_{n}\right) / k-\alpha\right| \geqq \nu\right) \leqq 4 \nu$ uniformly in $n$ and hence condition (3.3) is satisfied. $\sum_{k=1}^{\infty} P\left(X_{k} \geqq M\right)<\infty$ so that $P\left(\bigcap_{n=1}^{\infty} \bigcup_{k=n}^{\infty}\left\{X_{k} \geqq M\right\}\right)=0$. This is enough to establish condition (3.4).

Theorem 3. Let $\left\{X_{n}\right\}$ be a sequence of non-negative independent random variables. If (3.3) and (3.4) are satisfied and if either (2.3) or for some $n$ $(2.4-n)$ hold, then for any $h>0 \lim _{x \rightarrow \infty}[H(x)-H(x-h)]=h / \alpha$. If the random variables are integer valued, we ask that (3.3) and (3.4) hold and that $\lim _{n \rightarrow \infty}\left[u_{n}-u_{n-1}\right]=0$. Then $\lim _{n \rightarrow \infty} u_{n}=1 / \alpha$. 
Proof. The hypotheses of both Lemma 2-A and Lemma 3-A are satisfied so that for any $h>0$ and $\nu>0$ there exists $z^{\prime}>0$ such that for all $x$ and all integers $k \geqq z^{\prime} / h$,

$$
\begin{gathered}
\left|\sum_{v=1}^{k} \frac{[H(x+v h)-H(x+[v-1] h)]}{k h}-1 / \alpha\right| \\
=\left|\frac{H(x+k h)-H(x)}{k h}-1 / \alpha\right| \leqq \nu .
\end{gathered}
$$

If condition (2.3) is satisfied then the desired conclusion, (3.1), follows immediately. If the random variables are integer valued and

$$
\lim _{n \rightarrow \infty}\left(u_{n}-u_{n-1}\right)=0
$$

then (3.2) can be obtained directly from the above inequality by setting $h=1$.

We consider next the case where for some $n,(2.4-n)$ holds. Take $\nu$ so that $0<\nu<h / 2$. Let $I_{h}$ be the indicated function of the interval $(0, h]$ and $J_{h}$ be that of $(\nu, h-\nu]$. Let $g(x)$ have $n+3$ continuous derivatives and be such that $J_{h}(x) \leqq g(x) \leqq I_{h}(x)$ for all $x$. For all $k \geqq z^{\prime} /(h-2 v)$ we have

$$
\begin{aligned}
g * \sum_{v=1}^{k}\left[\frac{H(x+v[h-2 \nu])}{k(h-2 \nu)}\right] & \geqq J_{h} *\left[\sum_{v=1}^{k} \frac{H(x+v[h-2 \nu])}{k(h-2 \nu)}\right] \\
& =\frac{H(r-\nu+k[h-2 \nu])-H(x-\nu)}{k(h-2 \nu)} \geqq 1 / \alpha-\nu .
\end{aligned}
$$

This inequality holds for all $x . I_{h} * H(x)$ is uniformly bounded in $x$ so that there exists $\left\{x_{j}\right\}$ and a finite number, $m$, for which $\lim _{x \rightarrow \infty} \inf I_{h} * H(x)=$ $\lim _{j \rightarrow \infty} I_{h} * H\left(x_{j}\right)=m$. By condition $(2.4-n)$ there exists $x^{\prime}$ such that for all $x_{j} \geqq x^{\prime}$ and all $v, 1 \leqq v \leqq k, g * H\left(x_{j}+v[h-2 \nu]\right) \leqq m+\nu$. Therefore $k(m+\nu) / k(h-2 \nu) \geqq 1 / \alpha-\nu$ or $m \geqq(h-2 \nu)(1 / \alpha-\nu)-\nu$. Next let $E_{h}$ be the indicator function of the interval $(-\nu, h+\nu]$ and choose $g^{\prime}$ so that $g^{\prime}$ has $n+3$ continuous derivatives and $I_{h}(x) \leqq g^{\prime}(x) \leqq E_{h}(x)$. Then

$$
\begin{aligned}
g^{\prime} *\left[\sum_{v=1}^{k} \frac{H(x+v[h+2 \nu])}{k(h+2 \nu)}\right] \\
\leqq E_{h} *\left[\sum_{v=1}^{k} \frac{H(x+v[h+2 \nu])}{k(h+2 \nu)}\right] \\
=\frac{H(x+\nu+k[h+2 \nu])-H(x+\nu)}{k(h+2 \nu)} \leqq 1 / \alpha+\nu .
\end{aligned}
$$

Repeating the above argument yields for $\limsup _{x \rightarrow \infty} I_{h} * H(x)=M, M$ $\leqq(h+2 \nu)(1 / \alpha+\nu)+\nu$. But $\nu$ is arbitrary so $M=m=h / \alpha$. 
Next we consider the case when $\alpha$ is infinite.

Corollary 3-A. Let $\left\{X_{n}\right\}$ be a sequence of independent non-negative random variables with

$$
\frac{S_{n+k}-S_{n}}{k}
$$

converging in probability to infinity as $k$ approaches infinity uniformly in $n$.

If there exists for some $d$ for which

$$
\lim _{x \rightarrow \infty}([H(x+d)-H(x)]-[H(x)-H(x-d)])=0,
$$

or if for some $n, n \geqq 0$, and any $g$ with compact support and $n+3$ continuous derivatives $\lim _{x \rightarrow \infty} g *[H(x+d)-H(x)]=0$, then for any $h>0$, $\lim _{x \rightarrow \infty}[H(x+h)-H(x)]=0$. If the random variables are integer valued and if for some integer $d, \lim _{n \rightarrow \infty}\left(u_{n}-u_{n-d}\right)=0$, then $\lim _{n \rightarrow \infty} u_{n}=0$.

Proof. From the assumption (3.5) we have that for any $\nu>0$ there exists $k^{\prime}$ such that for all $k \geqq k^{\prime}$ and all $n, P\left(\left(S_{n+k}-S_{n}\right) / k \leqq 1 / \nu\right) \leqq \nu$. The argument of Lemma 2-A gives a $z^{\prime}$ such that for all $z \geqq z^{\prime}$ and all $j, H_{j}(z) / z \leqq \nu$. Consequently there exists $z^{\prime \prime}$ such that for all $z \geqq z^{\prime \prime}$ and all $x,(H(x+z)-H(x)) / z \leqq \nu$. Using the inequalities and the extimates found in Theorem 3 , we have that in the continuous case

$$
\limsup _{x \rightarrow \infty}[H(x+d)-H(x)]=0=\lim _{x \rightarrow \infty}[H(x+d)-H(x)] .
$$

If $h<d$ then $H(x+h)-H(x) \leqq H(x+d)-H(x)$ and hence we have the desired result. If $h>d$ we can write

$$
H(x+h)-H(x)=\sum_{v=1}^{k}\left[H\left(x+v h^{\prime}\right)-H\left(x+[v-1] h^{\prime}\right)\right]
$$

where $h=k h^{\prime}$ and $h^{\prime}<d$. The conclusion for the integer valued case follows by letting $h=1$ and restricting $x$ and $d$ to the set of positive integers.

REMARK. For a continuous renewal sequence, the hypotheses of Corollary 3-A, except in the situation described in Corollary 2-C, seem no easier to verify than either (2.3) or, for some $n,(2.4-n)$. However, if the variables are integer valued, this is not the case. Corollary 1-C gives conditions which insure that for some integer $d, \lim _{n \rightarrow \infty}\left(u_{n}-u_{n-d}\right)=0$. These same conditions do not imply that $\lim _{n \rightarrow \infty}\left(u_{n}-u_{n-1}\right)=0$.

REMARK. The condition (3.5) of Corollary 3-A will be satisfied if the non-negative independent variables are identically distributed with infinite first moments. If for some $N,\left(\left(S_{n+k}-S_{n}\right)-E\left(S_{n+k}-S_{n}\right)\right) / k$ converges in probability to 0 as $k$ approaches infinity uniformly for all $n \geqq N$ and if $\lim _{k \rightarrow \infty} \sum_{j=1}^{k} E\left(X_{n+j}\right) / k=\infty$ uniformly in $n$, then (3.5) is also satisfied.

We conclude this section with the following special corollary. 
Corollary 3-B. Let $\left\{X_{n}\right\}$ be a sequence of non-negative, independent, integer valued, random variables. If there exist a subsequence, $\left\{X_{n_{k}}\right\}$ of $\left\{X_{n}\right\}$, a sequence $\left\{a_{k}\right\}$ of real numbers, positive constants $M, \omega, \alpha$, and $N$, and relatively prime integers $m$ and $d$ such that conditions (i) through (iv) below are satisfied, then $\lim _{n \rightarrow \infty} u_{n}=1 / \alpha$.

(i) For all $k, P\left(\left|X_{n_{k}}-a_{k}\right|<M\right) \geqq \omega$.

(ii) For any $0<r<\pi / d$, there exists $u>0, u=u(r)$, such that for all $T$ satisfying $r \leqq|t| \leqq \pi / d$ we have $\sup _{k \geqq 1}\left|\rho_{k}(t)\right| \leqq 1-u$. Here $\rho_{k}(t)$ is defined as in Theorem 1.

(iii) for all $n \geqq N, \sum_{j=0}^{\infty} P\left(X_{n}=m+j d\right)=1$.

(iv) Conditions (3.3) and (3.4) are satisfied.

Proof. In the notation of Theorem 1 we can write

$$
u_{n}=p_{n}(1)+p_{n}(2)+\cdots+p_{n}(k-1)+\sum_{j=k}^{\infty} p_{j}(k) u_{n-j}(k) .
$$

Therefore the problem reduces to that of showing that the corollary is true in the case $N=1$. For $v$ a fixed integer satisfying $0 \leqq v<d$, let $Y_{k}=X_{k d+v}$ $+X_{k d+v-1}+\cdots+X_{(k-1) d+v+1}$ when $k>0$ and $Y_{0}=X_{1}+\cdots+X_{v}$. Here it is understood that when $v=0, Y_{0}=0$. Let $T_{n}=\sum_{k=1}^{n} Y_{k}$. Then we can write $u_{n d+m v}=\sum_{j=0}^{n} P\left(T_{j}=m v+n d\right)$. Conditions (i) and (ii) yield, by Corollary $1-\mathrm{C}, \lim _{n \rightarrow \infty}\left(u_{m v+n d}-u_{m v+(n-1) d}\right)=0$. For any $\nu>0$,

$$
P\left(\left|\frac{T_{n+j}-T_{j}}{n}-d \alpha\right| \geqq \nu\right)=P\left(\left|\frac{S_{v+d(n+j)}-S_{v+d j}}{d n}-\alpha\right| \geqq \nu / d\right)
$$

so that condition (3.3) is satisfied for $\left\{Y_{n}\right\}$ with the constant equal to $d \alpha$. Next let

$$
D_{k}(x, z)=\bigcap_{k=1}^{\infty}\left\{\left[\sum_{j=1}^{k} Y_{j}<x\right] \cup\left[\sum_{j=1}^{k} Y_{j} \geqq x+z\right]\right\} .
$$

Then $D(x, z) \leqq \bigcup_{k=0}^{d-1} A(x+k z / d, z / D)$. Then (3.4) is also satisfied for $\left\{Y_{n}\right\}$. Hence for any $\nu>0$ and all large $k$,

$$
\left|\sum_{j=1}^{k} \frac{u_{m v+(j+n) d}}{k d}-\frac{1}{d \alpha}\right| \leqq \nu
$$

uniformly in $n$. Therefore we have $\lim _{n \rightarrow \infty} u_{m v+n d}=1 / \alpha, v=0,1, \cdots, d-1$. Finally, if $m$ and $d$ are relatively prime, then for every integer $k \geqq d^{2}$ there exists positive integers $n$ and $v, 0 \leqq v \leqq d-1$, such that $k=m v+n d$. This completes the proof.

\section{Examples.}

Example (4.1). Define a random variable $Y$ by $P(Y=1)=P(Y=3)$ $=1 / 2$, and a random variable $Z$ by $P(Z=2)=1$. Let $\left\{X_{n}\right\}$ be a sequence 
of independent random variables such that if $n=k(k-1)$ for some positive integer $k$ then $X_{n}={ }^{d} X_{n-1}={ }^{d} Y$. Otherwise $X_{n}={ }^{d} Z$. The largest value that $S_{k(k-1)}$ may assume is $3[2(k-1)]+2[k(k-1)-2 k+2]=2 k^{2}-2$. Following $X_{k(k-1)}$ is a run of $Z$ 's long enough to insure that for no $n>k(k-1)$ can $S_{n}=2 k^{2}-3$. If $n<k(k-1), \quad S_{n} \leqq 2 k^{2}-5$. Finally $S_{k(k-1)}$ must take on an even value and hence for all $k, u_{2 k^{2}-3}=0$. From this we see that $\lim _{n \rightarrow \infty}\left(u_{n}-u_{n-1}\right) \neq 0$. If it were 0 an application of Lemma 3-B and Theorem 3 would give $\lim _{n \rightarrow \infty} u_{n}=1 / 2$ and a contradiction. This example is of interest because if for all $n, X_{n}={ }^{d} Y$ then we would have $\lim _{n \rightarrow \infty}\left(u_{n}-u_{n-1}\right)=0$ as a consequence of Corollary 1-D.

Example (4.2). Define a random variable $Y$ by $P(Y=1)=P(Y=\sqrt{ } 2)$ $=1 / 2$, and a random variable $Z$ by $P(Z=\sqrt{ } 2-1)=1$. Let $\left\{X_{n}\right\}$ be a sequence of independent random variables such that if $n=k(k-1)$ for some positive integer $k$, then $X_{n}={ }^{d} Y$. Otherwise $X_{n}={ }^{d} Z$. Then there exists $\left\{x_{k}\right\}$ with $\lim _{k \rightarrow \infty} x_{k}=\infty$ such that for any $h, 0<h<\sqrt{ } 2-1$, $H\left(x_{k}+h\right)-H\left(x_{k}\right)=0$ for all $k$. We cannot have (2.3) for if (2.3) were satisfied then an appeal to Lemma 3-B and Theorem 3 would give $\lim _{x \rightarrow \infty}[H(x+h)-H(x)]=h /(\sqrt{ } 2-1)$ for any $h$. This is clearly impossible. However if for all $n X_{n}={ }^{d} Y$ then we could conclude from Corollary 4.2 that (2.3) held for $\left\{X_{n}\right\}$.

EXAmpLe (4.3). Let $\left\{X_{n}\right\}$ be a sequence of positive independent random variables. Let $\left\{X_{n_{k}}\right\}$ be a subsequence of $\left\{X_{n}\right\}$ such that if $k=2 j$ for some integer $j$ then $P\left(X_{n_{k}}=1\right)=P\left(X_{n_{k}}=3\right)=1 / 2$, and if $k=2 j-1$ for some integer $j$ then $P\left(X_{n_{k}}=1\right)=P\left(X_{n_{k}}=\sqrt{ } 2\right)=1 / 2$. If we write $\rho_{k}(t)=E\left(e^{\left.i t X_{n_{k}}\right)}\right.$, then $\left|\prod_{k=1}^{q} \rho_{k}(t)\right| \leqq\left\{(1 / 4)\left|e^{2 i t}+e^{i t\left(1+V^{2}\right)}+e^{4 i t}+e^{i t\left(3+V^{2}\right)}\right|\right\}^{[q / 2]}$. According to Lemma 2-B there exists $t^{\prime}$ such that for all $t,|t| \geqq t^{\prime}$, $(1 / 3)\left|e^{2 i t}+e^{i t(1+\sqrt{ } 2)}+e^{i t(3+\sqrt{ } 2)}\right| \leqq 1-1 / t^{3}$. Hence $|t| \geqq t^{\prime}$ implies $\prod_{k=1}^{q}\left|\rho_{k}(t)\right|$ $\leqq\left(1-3 / 4 t^{3}\right)^{\mid q / 2]}$. We can now make the same kind of estimates that were made in Corollary 2-B except for factors of 2 and $4 / 3$. Therefore we have that for $\left\{X_{n}\right\},(2.4-3)$ is satisfied. If in addition $\left\{X_{n}\right\}$ satisfies (3.3) and (3.4) then for any $h, \lim _{x \rightarrow \infty}[H(x+h)-H(x)]=h / \alpha$. The presence of either the subsequence $\left\{X_{n_{2 j}}\right\}$ or $\left\{X_{n_{2 j-1}}\right\}$ alone would not be enough to guarantee a limit in the above expression. However by mixing the two subsequences and looking closely at the proof of Corollary $2-\mathrm{B}$, we find that the subsequence $\left\{X_{n_{k}}\right\}$ will give us the condition (2.4-n) needed for the renewal theorem.

EXAMPLE (4.4). Let $\left\{X_{n}\right\}$ be a sequence of independent random variables whose distribution functions are defined as follows: For $0<x<1, P\left(X_{n}<x\right)$ $=x(1-1 / n \ln (3+n)) ; P\left(X_{n}=n\right)=1 / n[\ln (3+n)]$. In the notation of $\S 2$, the subsequence $\left\{X_{n_{k}}\right\}$ is the given $\left\{X_{n}\right\}, a_{k}=1 / 2, M=1 / 2$, and $\omega=1-1 / \ln 4$. From Corollary $2-A$ we see that (2.4-0) is satisfied. The remark following Corollary 2-A tells us that actually (2.3) holds for 
$\left\{X_{n}\right\}$. We have $\left\{E\left(X_{n}\right)\right\}$ monotone increasing with $\lim _{n \rightarrow \infty} E\left(X_{n}\right)=1 / 2$. Hence $\lim _{k \rightarrow \infty}(1 / k) \sum_{j=1}^{k} E\left(X_{n+j}\right)=1 / 2$ uniformly in $n$. If we let $G(x)$ $=\inf _{n \geq 1} P\left(X_{n}<x\right)$, then it can be verified that the conditions of Lemma 3-C are satisfied. Therefore we have (3.1). In this example the sequence, $\left\{X_{n}\right\}$, obeys the weak but not the strong law of large numbers. Sequences satisfying the conditions of Lemma 3-B will always obey the strong law of large numbers. This example also puts an end to any thought that from (3.1) it might be possible to prove that $P\left(\lim _{n \rightarrow \infty}(1 / n) \sum_{k=1}^{n} X_{k}=\alpha\right)=1$.

\section{BiBLIOGRAPHY}

1. D. Blackwell, A renewal theorem, Duke Math. J. 15 (1948), 145-151.

2. __ Extension of a renewal theorem, Pacific J. Math. (2) 3 (1953), 315-320.

3. Y.S. Chow and $\mathrm{H}$. Robbins, $A$ renewal theorem for random variables which are dependent or nonidentically distributed, Ann. Math. Statist. 34 (1963), 390-395.

4. K. L. Chung and J. Wolfowitz, On a limit theorem in renewal theory, Ann. of Math. (2) 55 (1952), 1-6.

5. J. L. Doob, Stochastic processes, Wiley, New York, 1953, p. 45.

6. P. Erdös, W. Feller and H. Pollard, A theorem on power series, Bull. Amer. Math. Soc. 55 (1949), 201-204.

7. W. Feller, On the integral equation of renewal theory, Ann. Math. Statist. 12 (1941), 243-267.

8. W. Feller and S. Orey, A renewal theorem, J. Math. Mech. 10 (1961), 619-624.

9. G. H. Hardy and E. M. Wright, Introduction to the theory of numbers, 4th ed., Clarendon Press, Oxford, 1960, p. 168.

10. T. Kawata, A renewal theorem, J. Math. Soc. Japan 8 (1956), 118-12

11. A. Kolmogorov, Anfangsgründer der Theorie der Markoffschen Ketten mit unendlich vielen möglichen Zuständen, Mat. Sb. (N.S.) 1 (1936), 607-610.

12. Walter L. Smith, On some general renewal theorems for nonidentically distributed variables, Fourth Berkeley Symposium on Mathematical Statistics and Probability, II, pp. 467-515, Univ. of California Press, Berkeley, Calif., 1961.

UNIVERSITY OF MINNESOTA, MinNEAPOLIS, MinNESOTA 